\title{
Stochastic Dominance and Risk Measure: A Decision-Theoretic Foundation for VaR and $\mathrm{C}-\mathrm{VaR}$
}

\author{
Chenghu Ma and Wing-Keung Wong*
}

November 26, 2006

\begin{abstract}
Is it possible to obtain an objective and quantifiable measure of risk backed up by choices made by some specific groups of rational investors? To answer this question, in this paper we establish some behavior foundations for various types of VaR models, including VaR and conditional-VaR, as measures of downside risk. Though supported to some extent with unanimous choices by some specific groups of expected or non-expected utility investors, VaRs as profiles of risk measures at various levels of risk tolerance are not quantifiable - they can only provide partial and incomplete risk assessments for risky prospects.

Also included in our discussion are the relevant VaRs and several alternative risk measures for investors; these alternatives use somewhat weaker assumptions about risk-averse behavior by incorporating a mean-preserving-spread. For this latter group of investors, we provide arguments for and against the standard deviation vs. VaR and conditional VaRs as objective and quantifiable measures of risk in the context of portfolio choice.

Key Words: downside risk, value-at-risk, conditional-VaR, stochastic dominance, utility

JEL Classification: C0, D81, G10

\footnotetext{
${ }^{*} \mathrm{Ma}$ is Chair Professor of Economics at Wang Yanan Institute for Studies in Economics (WISE), Xiamen University, China; and Wong is the Deputy Director, Risk Management Institute, National University of Singapore. We are grateful for support from both institutes. For correspondence, please e-mail to cma2006@xmu.edu.cn or to ecswwk@nus.edu.sg
} 


\section{Introduction}

No investors or portfolio managers will argue with the idea that downside risk is a major cause of stress and a factor to be taken into consideration in measuring financial risks. In the classical mean-variance (MV) context of Markowitz (1952), the variance or standard deviation is adopted to measure the risk exposure of financial assets and portfolios of financial assets. However, these measures fail to capture the downside risk of most risky prospects, particularly when the random payoffs are not normally distributed. To circumvent this problem, academics have proposed several measures. Among them, the most commonly used measures are the Value-at-Risk (VaR) and the conditional-VaR, or simply, C-VaR. ${ }^{1}$ Jorion (2000) and Alexander and Baptista $(2002,2004)$ are advocates of the former while Rockafellar and Uryasev $(2000,2002)$ are promoters of the latter. Since J.P. Morgan adopted VaR in its RiskMetrics System, both VaR and C-VaR have become standard instruments for measuring risk in portfolio management.

In this paper, our objective is to provide a decision-theoretical foundation for $\mathrm{VaR}$ and conditional-VaR, and to examine the relevance of $\mathrm{VaR}$ and conditional-VaR to choices made by rational investors. ${ }^{2}$ For example, taking two risk prospects with random payoffs $X$ and $Y$, respectively, suppose $X$ and $Y$ involve the same level of initial investment, and suppose $X$ has a smaller $\mathrm{VaR}$ or $\mathrm{C}-\mathrm{VaR}$ than $Y$ at various levels of risk tolerance. The questions we ask are:

\section{Problem}

(i) Would rational investors or a specific group of rational investors prefer $X$ to $Y$ ?

(ii) Or the converse, if rational investors or a specific group of rational investors prefer $X$ to $Y$, do they regard $Y$ as being riskier than $X$ based on the criterion of VaR?

\footnotetext{
${ }^{1}$ As first noted by Acerbi and Tasche (2002), there are various ways to define VaR and conditional VaR when random payoffs are with discontinuous c.d.f.s. The notions of VaR and conditional VaR proposed in this paper can be expressed by making use of the leftrather than the right-continuously inverse function of the c.d.f. We provide arguments in support of this treatment (see Section 4.1).

${ }^{2}$ Unless specified otherwise, in this paper, the notion of a rational investor refers to an expected-utility investor whose behavior under risk fulfills the von Neumann and Morgenstern (1944) axioms even though much of the analysis developed in this paper also applies to investors with non-expected utility functions.
} 
Affirmative answers to these sorts of questions are critical in establishing $\mathrm{VaR}$ and $\mathrm{C}-\mathrm{VaR}$ as objective risk measures. On the one hand, for practical implementation, it is always desirable to treat risk objectively, and it is particularly desirable to obtain a quantifiable risk measure. ${ }^{3}$ Both VaRs and the standard deviation alike, which do not rely on investors' preferences and their risk attitudes, are regarded as objective measures.

On the other hand, one may argue that the assessment of risk is a subjective matter because people with different risk attitudes and financial status may reach different assessments of the risk involved in a common risky prospect. Also, though it is not difficult to obtain a quantifiable risk assessment (e.g., risk premium) at an individual level, it is difficult to imagine that a group of investors with possibly different attitudes toward risk will come up with a common figure with respect to risk assessment.

Thus, the possibility of obtaining an objective and quantifiable risk measure can only be discussed within a decision-theoretical context. To obtain an objective and quantifiable risk measure from various subjective risk assessments, there must be unanimous agreement on risk assessments among some specific groups of investors $\mathcal{I}$. This leads us to define an objective risk measure as a real-valued function $X \rightarrow \rho(X)$ over a set of random payoffs $\mathbb{X}$ (involving the same level of initial investment) to be such that, for all $X, Y \in \mathbb{X}$,

$$
\rho(X) \leq \rho(Y) \text { if, and only if, all risk-averse investors in } \mathcal{I} \text { prefer } X \text { to } Y .
$$

The above definition of a risk measure suggests that when a unanimous ranking of some risk perspectives is reached among a specific group of investors, and when such ranking conforms to a pre-specified objective and quantifiable risk measure, one may say that the underlying objective measure represents risk assessments made by the group of investors. In this case, when the choice made is found to be consistent with the specific risk measure in the above sense, then one may say that "investors' choice of $X$ over $Y$ is due to $Y$ being riskier than $X$ as indicated by the risk measure $\rho(\cdot) . "$

The major difficulty in obtaining an objective and quantifiable measure from subjective risk assessments lies in the fact that it is not always pos-

\footnotetext{
${ }^{3}$ See below for the precise definition of a 'quantifiable' risk measure. The VaR or conditional VaR is quantifiable at any arbitrary given level of risk tolerance, but the value varies with the level of risk tolerance. So, investors with different levels of risk tolerance may end up with different risk assessments. In this sense we say that the VaR or conditional VaR corresponds to a profile of risk measure, which itself does not constitute a quantifiable measure.
} 
sible to obtain a unanimous agreement among a specific group of investors concerning the risk measure (if any) in guiding their choices. We may think of using a risk premium in the context of Arrow (1970) and Pratt (1964). As a quantifiable risk measure (for individual investors), the risk premium varies with investors' risk attitudes and with their underlying utility functions. Since different investors may end up paying different premiums when facing a common risk prospect, it is difficult to imagine how these investors could agree upon a specific common risk measure.

There is another conceptual difficulty encountered when trying to obtain an objective measure of risk. One may argue that risk assessments are implicitly or indirectly embedded in the actions taken and choices made by the investors - it is not always possible to tell from their choices or actions which risk measures the investors use to assess the risk, let alone if all investors concerned agree upon a specific common objective risk measure.

Indeed, the message in this paper, to those who wish to obtain an objective and quantifiable measure of risk, is a negative one. Thus, with respect to some specific groups of expected-utility investors, we show that it is impossible to obtain an objective and quantifiable risk measure $\rho(\cdot)$ to represent choices made by the group. Nevertheless, there exist some partial or incomplete binary relations (in the form of lexicographic utility functions), referring to VaR and C-VaR at all levels of risk tolerance, to represent investors' assessment of risk. For instance, as illustrated below, both VaR and conditional-VaR criteria (at all levels of risk tolerance) constitute (incomplete) risk measures for some specific groups of expected-utility investors.

Stochastic dominance (SD), using different partial orders defined over a set of risky payoffs, provides a useful criterion for portfolio choice and risk measurement. Originating from majorization theory (Hardy, Littlewood, and Pólya, 1934; Marshall and Olkin 1979), stochastic dominance was formally developed by Quirk and Saposnik (1962), Hadar and Russell (1969), Hanoch and Levy (1969), and Rothschild and Stiglitz (1970). The SD approach has been regarded as one of the most useful tools for ranking investment prospects when there is uncertainty (see, for example, Levy 1992). Similar to a mean-variance criterion, the SD was used long before VaR was introduced in the late 1980s. The rationale for the stochastic dominance criterion has been well defined (Rothschild and Stiglitz 1970) as follows:

$X$ dominates $Y$ by the first-order (respectively, second-order) stochastic dominance if and only if all investors who prefer more to less (respectively, be risk-averse) would prefer $X$ to $Y$.

Efforts to find an (objective) risk measure consistent with the SD crite- 
rion have been made in the literature. For instance, Ogryczak and Ruszczyński (2002) define an objective risk measure $r$ to be consistent with SSD by

$$
X \text { dominate } Y \text { by SSD } \Rightarrow E[X] \geq E[Y] \text { and } r_{X} \leq r_{Y} \text {. }
$$

Note that a risk measure $r$ that is consistent with SSD in the sense of Ogryczak and Ruszczyński (2002) may not provide a unanimous assessment of risk among risk-averse expected-utility investors. In fact, there are many risk measures that are consistent with SSD and choices made by risk-averse expected-utility investors in the sense of Ogryczak and Ruszczyński (2002), but none of these are 'consistent' according to our definition. To put it another way, even if $X$ is considered less risky than $Y$ in the sense of $E[X] \geq$ $E[Y]$ and $r_{X} \leq r_{Y}$, it is always possible to find expected-utility investors who strictly prefer $Y$ to $X$. For that reason we say that the notion of 'consistency' adopted in this paper is stronger than the notion of a 'consistent risk measure' introduced by Ogryczak and Ruszczyński (2002).

Thus, this paper's main contribution is to establish some logical connections between these two seemingly very different investment criteria: one concerned with risk exposure, and the other concerned with stochastic dominance. As the latter is known to be equivalent to unanimous choices made by expected-utility investors, it is interesting to note that the VaR criterion, as a profile of quantitative measures, is equivalent to the first-order stochastic dominance (FSD). This finding was first reported in Ogryczak and Ruszczyński (2002, Remark 2). In this paper, we also propose two different notions of a conditional VaR, namely, C-VaR and c*-VaR. The $\mathrm{c}^{*}$-VaR, as a profile of downside risk measures at various levels of risk tolerance, is shown to be equivalent to the second-order stochastic dominance (SSD). These add to the literature by establishing several useful, logical relationships among C-VaR, SSD, and choices made by risk-averse expected-utility investors.

The message resulting from these exercises is clear - it is impossible to obtain an objective and quantifiable risk measure that fully reflects choices made by the class of expected-utility investors. The implication of this for portfolio risk management and risk measurement is evident - one must think twice before seeking quantifiable risk measures as a guide to risk management and risk measurement. Risk assessment is best regarded as a subjective matter, and we shall leave it to individual investors and fund managers to decide for themselves which risk measure is most sensible in guiding their choices. A sensible risk measure is one that best reflects investors' risk attitudes and their preferences.

Having said that, as a minor point, we need to point out that the conditional VaR measures, namely, C-VaR and $c^{*}-\mathrm{VaR}$, proposed in this pa- 
per, differ from those in the existing literature, for example, the TVaR of Ogryczak and Ruszczyński (2002), and the AV@R of Leitner (2005), among others. Ogryczak and Ruszczyński (2002) established the equivalence between TVaR and the second-order stochastic dominance, and Leitner (2005) showed that AV@R as a profile of risk measures is equivalent to the SSD under certain conditions. The C-VaR introduced in this paper is defined as how much risk is expected to fall below the VaR measure. The VaR can be expressed alternatively by using the left, rather than the right, continuous inverse c.d.f. Not surprisingly, the $\mathrm{C}-\mathrm{VaR}$ as a profile of risk measure may not lead to the same assessment as the SSD criterion, particularly for random payoffs with discrete supports. In this paper we provide sufficient conditions under which the C-VaR and SSD are equivalent.

As a separate effort, we raise the issue of the relevance of VaR and conditional VaR as objective (non-quantifiable) risk measures for investors whose behavior may not necessarily conform to von Neumann and Morgenstern's (1944) expected utility axiom, not even to the broader class of SSDconforming non-expected utility functions. ${ }^{4}$ Several thought experiments are conducted in exploring the extent to which VaR, C-VaR, or $c^{*}-\mathrm{VaR}$ could serve as sensible risk measures. Along this line, we have developed a linkage between choices made by the class of investors who display meanpreserving-spread (MPS) risk aversion and the corresponding risk measure. ${ }^{5}$

According to Boyle and Ma (2004), SSD implies but is not implied by MPS. Also keeping in mind that SSD corresponds to unanimous choices made by risk-averse expected-utility investors (Rothschild and Stiglitz 1970), the MPS and SSD as different investment criteria would, in general, lead to different assessments of financial risk. But, as illustrated in Section 4.3 of this paper, we find that the standard deviation, as a measure of risk, will never contradict choices made by MPS-risk-averse investors.

Our assertion of the logical connection between the standard deviation and choices made by MPS-risk-averse investors is built on an earlier observation made by Boyle and Ma (2004) in the context of portfolio choices. They show that all MPS-risk-averse investors would optimally invest along Markowitz's classical efficient frontier. Accordingly, we shall not be able to construct a portfolio that achieves the expected return at the optimal level,

\footnotetext{
${ }^{4} \mathrm{~A}$ utility function $u$ on $\mathbb{X}$ is said to be 'SSD- conforming' if $u(X) \geq u(Y)$ whenever $X$ dominates $Y$ by the SSD.

${ }^{5}$ The notions of MPS and strong-MPS are defined below in Section 4.3. The MPS refers to that of Boyle and Ma (2004), while the strong-MPS refers to the 'mean-preservingspread' adopted by Rothschild and Stiglitz (1970). The strong MPS implies MPS but the converse does not hold.
} 
even though it has a smaller 'risk' when measured by the standard deviation. In this sense we may say that the standard deviation is an objective measure consistent with the MPS-risk-averse behavior assumption.

The remainder of the paper is organized as follows. In Section 2, we introduce and study three objective measures of downside risk associated with risky payoffs. These are VaR, conditional-VaR, and a modified conditional$\mathrm{VaR}$. The last two risk measures are respectively denoted by $\mathrm{C}-\mathrm{VaR}$ and $c^{*}$-VaR. The decision-theoretical foundations of these objective measures of risk and their useful links with the notions of stochastic dominance are provided in Section 3. We will show that VaR is equivalent to the first-order stochastic dominance, while $\mathrm{c}^{*}-\mathrm{VaR}$ and $\mathrm{C}-\mathrm{VaR}$ are linked to the second-order stochastic dominance. Section 4 contains a discussion of the advantages and limitations of VaR and C-VaR as risk measures, and their comparison with some alternative (existing or new) risk measures such as the standard deviation. Section 5 summarizes the paper. Some of the mathematical proofs are summarized in the Appendix.

\section{Various VaR Models}

In this section, we introduce various types of VaR risk measures, including VaR, C-VaR, and $\mathrm{c}^{*}$-VaR, and develop some basic properties of these measures.

\section{$2.1 \quad$ VaR and C-VaR}

Roughly speaking, VaR and C-VaR introduced below constitute two specific ways to measure the potential loss associated with a risky position on a predefined horizon, and the chance of such a loss is pre-specified at an arbitrary level of risk tolerance measured by the tail probability $\alpha \in(0,1]$. Precisely,

Definition 1 For any given $\alpha \in(0,1]$,

1. the value at risk, denoted by $\operatorname{VaR}(\alpha)$, for a risky project with random payoff $W$ is defined as the 'critical value' at which the probability of incurring a loss of no less than $\operatorname{VaR}(\alpha)$ is at least $\alpha$. That is,

$$
\operatorname{VaR}(\alpha)=\sup \left\{u: \operatorname{Pr}\left\{W \leq W_{0}-u\right\} \geq \alpha\right\}, \forall \alpha \in(0,1]
$$

where $W_{0}$ is the initial investment in the risky project. 
2. The conditional-VaR, or simply $C$-VaR, is defined accordingly by setting

$$
C-\operatorname{VaR}(\alpha) \triangleq E\left[W_{0}-W \mid W_{0}-W \geq \operatorname{VaR}(\alpha)\right], \forall \alpha \in(0,1] .
$$

Let $F_{W}(x) \triangleq \operatorname{Pr}\{W \leq x\}, x \in \mathbb{R}$, be the cumulative distribution function (c.d.f.) for $W$. The c.d.f. $F_{W}(\cdot)$ is known to be right-continuous and monotonically increasing. We may also introduce the left- and the rightcontinuously inverse functions, $\zeta_{W}^{-}(\alpha)$ and $\zeta_{W}^{+}(\alpha)$, of the c.d.f. with

$$
\begin{gathered}
\zeta_{W}^{-}(\alpha) \triangleq \inf \left\{x: F_{W}(x) \geq \alpha\right\} \\
\zeta_{W}^{+}(\alpha) \triangleq \inf \left\{x: F_{W}(x)>\alpha\right\}
\end{gathered}
$$

$\forall \alpha \in(0,1] .{ }^{6}$ The properties of left- and right-continuously inverse c.d.f. functions, $\zeta_{W}^{-}(\alpha)$ and $\zeta_{W}^{+}(\alpha)$, are summarized in the Appendix.

Various versions of VaRs and conditional VaRs could be introduced. Acerbi and Tasche (2002) provide a list of different expected shortfalls that link closely with the VaR and C-VaR introduced in our paper. But special care must be taken concerning the precise mathematical definition with respect to any specific measure of risk. For instance, we could use either a left- or right-continuously inverse function to define VaR. For atomless r.v.s with continuum supports, the two inverse functions coincide and will thus result in the same measure of value-at-risk. But for general r.v.s with possibly discrete supports, these two inverse functions will result in different assessments of value-at-risk. The same could be said with respect to C-VaR.

Furthermore, the notion of CVaR introduced by Uryasev (2000) and Pflug (2000), which is re-formulated in Acerbi and Tasche (2002, Definition $2.5)$, differs from our C-VaR. The two notions of conditional VaR coincide when the random payoffs are atomless with continuum supports. But the CVaR differs from the C-VaR for discrete random payoffs. The case with discrete supports is of practical value for empirical implementation. The same can be said with respect to the difference between the notion of the 'expected shortfalls' (ES) of Acerbi and Tasche (2002) and the C-VaR adopted in this paper. The former is equivalent to the CVaR, according to Acerbi and Tasche (2002, Corollary 4.3).

Incidently, in a recent paper by Leitner (2005), the right-continuously inverse function is used to define the so-called 'average value at risk,' or,

\footnotetext{
${ }^{6}$ The right-continuous function $\zeta_{W}^{+}$admits an alternative expression as $\zeta_{W}^{+}(\alpha)=$ $\sup \left\{x: F_{W}(x) \leq \alpha\right\}$.
} 
using his notation, AV@R. For general r.v.s with discrete supports, the AV@R differs from the C-VaR considered in this paper. In Section 4.1 below, we provide a justification for using the left- rather than the rightcontinuously inverse c.d.f. to define value-at-risk and conditional value-atrisk.

Keeping in mind the remarks above, we could readily express the VaR and the C-VaR by making use of the left-continuously inverse function $\zeta_{W}^{-}(\cdot)$ :

Proposition 1 Consider an integrable random payoff $W$ with initial investment $W_{0}$. We have:

1. $\operatorname{VaR}(\alpha)=W_{0}-\zeta_{W}^{-}(\alpha), \forall \alpha \in(0,1]$.

2. $C$ - $\operatorname{VaR}(\alpha)=g_{\alpha}(\operatorname{VaR}(\alpha)), \forall \alpha \in(0,1]$, where $x \rightarrow g_{\alpha}(x)$ is defined by setting

$$
g_{\alpha}(x) \triangleq x+\frac{1}{F_{W}\left(\zeta_{W}^{-}(\alpha)\right)} \int_{-\infty}^{W_{0}-x} F_{W}(t) d t .
$$

3. $C-\operatorname{VaR}(\alpha)=\min _{x \in \mathbb{R}} g_{\alpha}(x)$ and $\operatorname{VaR}(\alpha)=\max \left\{\arg \min _{x \in \mathbb{R}} g_{\alpha}(x)\right\}$.

Proof. The first statement follows by the definitions of the VaR and the left-continuously inverse c.d.f., we have:

$$
\begin{aligned}
\operatorname{VaR}(\alpha) & =\sup \left\{u: \operatorname{Pr}\left\{W \leq W_{0}-u\right\} \geq \alpha\right\} \\
& =W_{0}-\inf \{x: \operatorname{Pr}\{W \leq x\} \geq \alpha\} \\
& =W_{0}-\inf \left\{x: F_{W}(x) \geq \alpha\right\} \\
& =W_{0}-\zeta_{W}^{-}(\alpha)
\end{aligned}
$$

To prove statement 2 of the proposition, we have, for all $\alpha \in(0,1]$,

$$
\begin{aligned}
& \mathrm{C}-\operatorname{VaR}(\alpha) \\
= & \operatorname{VaR}(\alpha)+E\left[W_{0}-W-\operatorname{VaR}(\alpha) \mid W_{0}-W \geq \operatorname{VaR}(\alpha)\right] \\
= & \operatorname{VaR}(\alpha)+\frac{E\left[\left(W_{0}-\operatorname{VaR}(\alpha)-W\right)^{+}\right]}{F_{W}\left(W_{0}-\operatorname{VaR}(\alpha)\right)} \\
= & \operatorname{VaR}(\alpha)+\frac{1}{F_{W}\left(\zeta_{W}^{-}(\alpha)\right)} \int_{-\infty}^{W_{0}-\operatorname{VaR}(\alpha)} F_{W}(x) d x \\
= & g_{\alpha}(\operatorname{VaR}(\alpha))
\end{aligned}
$$

in which the third equality is obtained by setting $\operatorname{VaR}(\alpha)=W_{0}-\zeta_{W}^{-}(\alpha)$. 
Now, we proceed to prove the third statement of the proposition. First, for all $x \leq \operatorname{VaR}(\alpha)=W_{0}-\zeta_{W}^{-}(\alpha)$, by monotonicity of the c.d.f., we have: $F_{W}\left(W_{0}-x\right) \geq F_{W}\left(\zeta_{W}^{-}(\alpha)\right)$. This, in turn, implies

$$
g_{\alpha}^{\prime}(x)=1-\frac{F_{W}\left(W_{0}-x\right)}{F_{W}\left(\zeta_{W}^{-}(\alpha)\right)} \leq 0
$$

for all $x \leq \operatorname{VaR}(\alpha)$. Second, for all $x>\operatorname{VaR}(\alpha)$, by the definition of $\operatorname{VaR}$, we have:

$$
x>\operatorname{VaR}(\alpha) \Leftrightarrow x>\sup \left\{u: F_{W}\left(W_{0}-u\right) \geq \alpha\right\} .
$$

Since $x \notin\left\{u: F_{W}\left(W_{0}-u\right) \geq \alpha\right\}$, we have $F_{W}\left(W_{0}-x\right)<\alpha$. This, together with Lemma 1-(a), implies

$$
F_{W}\left(W_{0}-x\right)<\alpha \leq F_{W}\left(\zeta_{W}^{-}(\alpha)\right) .
$$

So, we have:

$$
g_{\alpha}^{\prime}(x)>0 \text { for all } x>\operatorname{VaR}(\alpha) .
$$

Combining the two inequalities in (6) and (7) for $g_{\alpha}^{\prime}(\cdot)$, one could easily show that $g_{\alpha}(\cdot)$ is monotonically decreasing from the left of $\operatorname{VaR}(\alpha)$ and is strictly increasing to the right of $\operatorname{VaR}(\alpha)$. So, we conclude that $\operatorname{VaR}(\alpha)=$ $\max \left\{\arg \min _{x \in \mathbb{R}} g_{\alpha}(x)\right\}$. This, together with $\mathrm{C}-\operatorname{VaR}(\alpha)=g_{\alpha}(\operatorname{VaR}(\alpha))$, yields $\mathrm{C}-\operatorname{VaR}(\alpha)=\min _{x \in \mathbb{R}} g_{\alpha}(x)$ as desired.

The following two remarks are in order here:

Remark 1 We may express $C$-VaR $(\alpha)$ explicitly in terms of the left-continuously inverse distribution function such that

$$
C-\operatorname{VaR}(\alpha)=W_{0}-\frac{1}{F_{W}\left(\zeta_{W}^{-}(\alpha)\right)} \int_{-\infty}^{\zeta_{W}^{-}(\alpha)} x d F_{W}(x)
$$

for all $\alpha \in(0,1]$. In particular, when the random payoff $W$ is atomless with continuum support, we have $F_{W}\left(\zeta_{W}^{-}(\alpha)\right)=\alpha$, and in this situation the $C$ - $\operatorname{VaR}(\alpha)$ admits the following simple expression:

$$
\begin{aligned}
C-\operatorname{VaR}(\alpha) & =W_{0}-\frac{1}{\alpha} \int_{-\infty}^{\zeta_{W}^{-}(\alpha)} x d F_{W}(x) \\
& =W_{0}-\frac{1}{\alpha} \int_{0}^{\alpha} \zeta_{W}^{-}(u) d u
\end{aligned}
$$

for all $\forall \alpha \in(0,1]$. So, for this particular special case of atomless r.v.s with continuum supports, the $C$-VaR reduces to Leitner's (2005) notion of AV@R. 
Remark 2 As another special case, we consider random payoff $W$ with finite or countable infinite support, that is,

$$
W=\left\{p_{1}, x_{1} ; \cdots ; p_{n}, x_{n} ; \cdots\right\}
$$

with $x_{1}<\cdots<x_{n} \cdots$. Let $C_{0}=0$ and $C_{n}=\sum_{i=1}^{n} p_{i}, n=1,2, \cdots$, be the corresponding cumulative probabilities. It holds true that

$$
\begin{aligned}
C-\operatorname{VaR}(\alpha) & =W_{0}-\frac{1}{C_{n+1}} \sum_{i=1}^{n+1} p_{i} x_{i} \\
\forall \alpha & \in\left(C_{n}, C_{n+1}\right], n=0,1, \cdots .
\end{aligned}
$$

\section{$2.2 \quad c^{*}-\mathrm{VaR}$}

This $c^{*}-V a R$ defined below as a measure of downside risk is arbitrary. Nevertheless, it coincides with the C-VaR for random payoffs with continuum supports. Moreover, in contrast to $\mathrm{VaR}$ and $\mathrm{C}-\mathrm{VaR}$, as illustrated below in Remark 5, the $\mathrm{c}^{*}$-VaR defined as a real function of risk tolerance $\alpha$ is continuous. In other words, $\mathrm{c}^{*}-\mathrm{VaR}$ as a measure of downside risk has the following desirable property: a small change in the level of risk tolerance will not cause a dramatic change in the assessment of downside risk. Indeed, it is difficult to imagine a risk measure function that would lead fund managers to act with ease when $\alpha=5 \%$ but be alarmed when $\alpha=4.999 \%$ !

Definition 2 For any integrable random payoff $W$, we define $c^{*}$-VaR as a measure of downside risk by setting

$$
c^{*}-\operatorname{VaR}(\alpha) \triangleq \operatorname{VaR}(\alpha)+\frac{1}{\alpha} \int_{-\infty}^{W_{0}-\operatorname{VaR}(\alpha)} F_{W}(x) d x
$$

for all $\alpha \in(0,1]$.

General relationships among VaR, C-VaR and $c^{*}$-VaR could be readily established and are summarized by the proposition below.

Proposition 2 Let $W$ be absolutely integrable. For all $\alpha \in(0,1]$ and $x \in \mathbb{R}$, we define $x \rightarrow h_{\alpha}(x)$ by setting

$$
h_{\alpha}(x) \triangleq x+\frac{1}{\alpha} \int_{-\infty}^{W_{0}-x} F_{W}(t) d t .
$$

Then, we have: 
(a) $\operatorname{VaR}(\alpha)=\max \left\{\arg \min _{x \in \mathbb{R}} h_{\alpha}(x)\right\}$ and $c^{*}-\operatorname{VaR}(\alpha)=\min _{x \in \mathbb{R}} h_{\alpha}(x)$;

(b) for all $\alpha \in(0,1], C-\operatorname{VaR}(\alpha) \leq c^{*}-\operatorname{VaR}(\alpha)$;

(c) if $W$ is with continuum support and atomless, then $c^{*}$ - $\operatorname{VaR}(\alpha)=C$ $\operatorname{VaR}(\alpha)$; and

(d) if $W$ is with discrete support, then, for all $\alpha \in\left(C_{n}, C_{n+1}\right], n=0,1, \cdots$, it holds true that

$$
c^{*}-\operatorname{VaR}(\alpha)=W_{0}-x_{n+1}+\frac{1}{\alpha} \sum_{i=1}^{n} C_{i}\left(x_{i+1}-x_{i}\right) .
$$

Proof. To prove (a), we first consider $x \leq \operatorname{VaR}(\alpha)=W_{0}-\zeta_{W}^{-}(\alpha)$. By Lemma 1-(a), we have $F_{W}\left(W_{0}-x\right) \geq F_{W}\left(\zeta_{W}^{-}(\alpha)\right) \geq \alpha$. With $h_{\alpha}^{\prime}(x)=$ $1-\frac{F_{W}\left(W_{0}-x\right)}{\alpha}, x \in \mathbb{R}$, we have $h_{\alpha}^{\prime}(x) \leq 0$ for all $x \leq \operatorname{VaR}(\alpha)$. On the other hand, by the definition of VaR, we have:

$$
x>\operatorname{VaR}(\alpha) \Leftrightarrow x>\sup \left\{u: F_{W}\left(W_{0}-u\right) \geq \alpha\right\} .
$$

Since $x \notin\left\{u: F_{W}\left(W_{0}-u\right) \geq \alpha\right\}$, we have $F_{W}\left(W_{0}-x\right)<\alpha$ or, equivalently, $h_{\alpha}^{\prime}(x)>0$ for all $x>\operatorname{VaR}(\alpha)$. Combining the two inequalities for $h_{\alpha}^{\prime}(\cdot)$, we conclude that $h_{\alpha}(\cdot)$ is monotonically decreasing from the left of $\operatorname{VaR}(\alpha)$ and is strictly increasing to the right of $\operatorname{VaR}(\alpha)$. This enables us to conclude that $\operatorname{VaR}(\alpha)=\max \left\{\arg \min _{x \in \mathbb{R}} h_{\alpha}(x)\right\}$. By the definition of $c^{*}-\operatorname{VaR}(\alpha)$, we obtain $\mathrm{c}^{*}-\operatorname{VaR}(\alpha)=\min _{x \in \mathbb{R}} h_{\alpha}(x)$.

For (b), by Lemma 1-(a), $g_{\alpha}(x) \leq h_{\alpha}(x)$ for all $\alpha \in(0,1]$ and $x \in \mathbb{R}$. Combining Proposition 1 and statement (a) proved above, we obtain

$$
\mathrm{C}-\operatorname{VaR}(\alpha)=\min _{x \in \mathbb{R}} g_{\alpha}(x) \leq \min _{x \in \mathbb{R}} h_{\alpha}(x)=\mathrm{c}^{*}-\operatorname{VaR}(\alpha) .
$$

For (c), when the random payoff $W$ is with continuum support and atomless, we have $F_{X}\left(\zeta_{W}^{-}(\alpha)\right) \equiv \alpha$ and $g_{\alpha}(\cdot)=h_{\alpha}(\cdot)$ on $(0,1]$. So, c*-VaR coincides with $\mathrm{C}-\mathrm{VaR}$. 
For (d), when $W$ has discrete support, the expression for the $\mathrm{c}^{*}-\mathrm{VaR}$ is obtained from (8) with $\zeta_{W}^{-}(\alpha)=C_{n+1}$ for $\alpha \in\left(C_{n}, C_{n+1}\right]$ and that

$$
\begin{aligned}
& \mathrm{c}^{*}-\operatorname{VaR}(\alpha) \\
= & W_{0}-x_{n+1}+\frac{1}{\alpha} \sum_{i=1}^{n} C_{i}\left(x_{i+1}-x_{i}\right) \\
\geq & W_{0}-x_{n+1}+\frac{1}{C_{n+1}} \sum_{i=1}^{n} C_{i}\left(x_{i+1}-x_{i}\right) \\
= & W_{0}-\frac{1}{C_{n+1}} \sum_{i=1}^{n+1} p_{i} x_{i} \\
= & \mathrm{C}-\operatorname{VaR}(\alpha)
\end{aligned}
$$

with equality only at $\alpha=C_{n+1}$.

Remark 3 Proposition 2-(a) provides a natural venue for finding VaR and $c^{*}-V a R$ by solving a static optimization problem. This, together with Proposition 1, enables one to compute VaR and $C$-VaR when analytic expressions are not readily available (for general distribution functions). One may follow a two-step procedure to compute $C$-VaR: In the first step, one may compute VaR by setting

$$
V a R=\max \left\{\arg \min _{x \in \mathbb{R}}\left\{x+\frac{1}{\alpha} \int_{-\infty}^{W_{0}-x} F_{W}(t) d t\right\}\right\} .
$$

An approximation of $V a R$ as the solution to the optimization problem could be obtained by following various numerical methods. With $\zeta_{W}^{-}=W_{0}-V a R$, in the second stage, we obtain (or approximation of) $C$-VaR by setting

$$
C-V a R=V a R+\frac{1}{F_{W}\left(W_{0}-V a R\right)} \int_{-\infty}^{W_{0}-V a R} F_{W}(t) d t .
$$

Remark 4 Proposition 2-(c) suggests that for random payoffs with discrete supports, for all $\alpha \in(0,1], c^{*}-\operatorname{VaR}(\alpha)$ must be strictly greater than $C$-VaR $(\alpha)$ except for $\alpha=C_{n}, n=1,2, \cdots$, at which it holds true that $c^{*}$ $\operatorname{VaR}\left(C_{n}\right)=C-\operatorname{VaR}\left(C_{n}\right)$.

Remark 5 As a separate observation, the function $\alpha \rightarrow c^{*}$-VaR $(\alpha)$ must be continuous on $(0,1]$. The assertion obviously holds true for random payoffs with continuum supports. To verify the validity of the assertion for 
discrete random payoffs, notice that $\alpha \rightarrow c^{*}-\operatorname{VaR}(\alpha)$ is piecewise continuous on $\left(C_{n}, C_{n+1}\right]$; moreover, at the boundary points, it is easy to see that

$$
\lim _{\alpha \rightarrow C_{n}} c^{*}-\operatorname{VaR}(\alpha)=c^{*}-\operatorname{VaR}\left(C_{n}\right)
$$

for all $n=1,2, \cdots$. We may thus conclude the continuity of the map $\alpha \rightarrow c^{*}$ $\operatorname{VaR}(\alpha)$ for all arbitrary random payoffs $W$.

\section{Value-at-Risk and Stochastic Dominance}

In this section, we show how the first-order and second-order stochastic dominances are linked to VaR, C-VaR, and $c^{*}$-VaR as measures of downside risk. Recall that $X$ is said to first-order stochastically dominate $Y$ if $F_{X}(x) \leq F_{Y}(x)$ for all $x \in \mathbb{R}$; and $X$ is said to second-order stochastically dominate $Y$ if

$$
\int_{-\infty}^{x}\left\{F_{X}(t)-F_{Y}(t)\right\} d t \leq 0 \text { for all } x \in \mathbb{R} .
$$

The first-order stochastic dominance is known for its equivalence for unanimous choices made by investors with monotonic expected utility functions, while the second-order stochastic dominance relationship is known for its equivalence for unanimous choices made by risk-averse expected-utility investors. ${ }^{7}$ For notational simplicity, we write $X \stackrel{\text { FSD }}{\succeq} Y$ and $X \stackrel{\text { SSD }}{\succeq} Y$ whenever $X$ dominates $Y$ according to FSD and SSD, respectively.

\subsection{FSD and VaRs}

Observe that if $X$ first-order stochastically dominates $Y$, then $X$ will involve less risk than $Y$ in the sense that it has a smaller probability to receive a payoff below any pre-specified critical value $x$. Therefore, $X$ must be associated with a smaller VaR than $Y$ if both $X$ and $Y$ require the same initial investment $W_{0}$. One could easily show that the converse of this observation is also true. We may thus put this formally as follows:

Theorem 1 For all $X$ and $Y$, we have

$$
X \stackrel{F S D}{\succeq} Y \Leftrightarrow \operatorname{VaR}_{X}(\alpha) \leq \operatorname{VaR}_{Y}(\alpha), \forall \alpha \in(0,1] .
$$

\footnotetext{
${ }^{7}$ Here, we assume that the improper integral in defining the SSD relationship takes finite values. Readers are referred to Wong and Ma (2006) for the precise conditions on the c.d.f.s of the random variables to ensure the validity of this last statement concerning the equivalence between SSD and choices by risk-averse expected-utility investors.
} 
Proof. Let $X \stackrel{\text { FSD }}{\succeq} Y$. For all $\alpha \in(0,1]$, we have:

$$
\alpha \leq F_{X}\left(\zeta_{X}^{-}(\alpha)\right) \leq F_{Y}\left(\zeta_{X}^{-}(\alpha)\right),
$$

where the first inequality follows by Lemma 1-(a), and the second inequality follows by the assumption of $X \stackrel{\text { FSD }}{\succeq} Y$. By the monotonicity of $\zeta_{Y}^{-}(\cdot)$, we obtain

$$
\zeta_{Y}^{-}(\alpha) \leq \zeta_{Y}^{-}\left(F_{Y}\left(\zeta_{X}^{-}(\alpha)\right)\right) \leq \zeta_{X}^{-}(\alpha)
$$

where the second inequality is obtained by Lemma 1-(b). This above inequality is equivalent to $\operatorname{VaR}_{X}(\alpha) \leq \operatorname{VaR}_{Y}(\alpha)$ as desired.

Conversely, let $\operatorname{VaR}_{X}(\alpha) \leq \operatorname{VaR}_{Y}(\alpha)$ for all $\alpha \in(0,1]$. Note that for all $x \in \mathbb{R}$ s.t. $F_{Y}(x)>0$, the value at risk for $X$ at $\alpha=F_{X}(x)$ is given by $\operatorname{VaR}_{X}\left(F_{X}(x)\right)=W_{0}-\zeta_{X}^{-}\left(F_{X}(x)\right)$. We have

$$
x \geq \zeta_{X}^{-}\left(F_{X}(x)\right) \geq \zeta_{Y}^{-}\left(F_{X}(x)\right),
$$

where the first inequality is given by Lemma 1-(b). By Lemma 1-(a) and by the monotonicity of $F_{X}(\cdot)$, we obtain

$$
F_{Y}(x) \geq F_{Y}\left(\zeta_{Y}^{-}\left(F_{X}(x)\right)\right) \geq F_{X}(x) .
$$

Since this is true for arbitrary $x \in \mathbb{R}$ in the support of $Y$, we thus conclude that $X \stackrel{\text { FSD }}{\succeq} Y$.

Remark 6 The observation on the equivalence between VaR and FSD as stated in Theorem 1 is fairly intuitive and is documented in Ogryzak and Ruszczynski (2002, Remark 2). We nevertheless choose to include this theorem, along with its proof, for the completeness of the exposition and for the convenience of our readers for future reference.

Remark 7 As another observation, Theorem 1 enables us to establish the following link between VaR, as a risk measure, and an anonymous choice by the group of expected-utility investors: For all $X$ and $Y$, we have

$$
\operatorname{VaR}_{X}(\alpha) \leq \operatorname{VaR}_{Y}(\alpha), \forall \alpha \in(0,1] \Leftrightarrow E[u(X)] \geq E[u(Y)]
$$

for all bounded and increasing utility functions $u \in \mathbb{C}(\mathbb{R})$.

From this remark, we observe that all expected-utility investors who prefer more to less will prefer $X$ to $Y$ whenever $Y$ is assessed to be riskier than $X$, according to the VaR criterion. But in order to assess risk between 
$X$ and $Y$, it has to pass the VaR test at all levels of risk tolerance $\alpha$. So, in general, the VaR criterion may not be able to perform the pair-wise risk assessments for any arbitrary given pair of risky prospects.

In addition to what is stated in Theorem 1, we establish, as follows, a couple of additional observations on FSD concerning its relationship with the other two measures of downside risk, namely, the $\mathrm{C}-\mathrm{VaR}$ and $\mathrm{c}^{*}-\mathrm{VaR}$ :

Corollary 1 For all $X$ and $Y$ that are absolute integrable, it must hold true that

1. $X \stackrel{F S D}{\succeq} Y \Rightarrow c^{*}-V_{a} R_{X}(\alpha) \leq c^{*}-V_{a} R_{Y}(\alpha), \forall \alpha \in(0,1]$.

2. If $Y$ is atomless and has continuum support, then

$$
X \stackrel{F S D}{\succeq} Y \Rightarrow C-\operatorname{VaR}_{X}(\alpha) \leq C-\operatorname{VaR}_{Y}(\alpha), \forall \alpha \in(0,1] .
$$

Proof. Suppose $X \stackrel{\text { FSD }}{\succeq} Y$, that is, $F_{X}(x) \leq F_{Y}(x), \forall x \in \mathbb{R}$. This, together with Proposition 2, implies that

$$
\begin{aligned}
& \mathrm{c}^{*}-\operatorname{VaR}_{X}(\alpha) \\
= & \min _{x \in \mathbb{R}}\left\{x+\frac{1}{\alpha} \int_{-\infty}^{W_{0}-x} F_{X}(t) d t\right\} \\
\leq & \min _{x \in \mathbb{R}}\left\{x+\frac{1}{\alpha} \int_{-\infty}^{W_{0}-x} F_{Y}(t) d t\right\} \\
= & \mathrm{c}^{*}-\operatorname{VaR}_{Y}(\alpha) .
\end{aligned}
$$

This proves the validity of the first statement.

To see the validity of the second statement, notice that for $Y$ with continuum support and atomless, it always holds true that $F_{Y}\left(\zeta_{Y}^{-}(\alpha)\right)=\alpha$. We have:

$$
\begin{aligned}
& \mathrm{C}-\operatorname{VaR}_{X}(\alpha) \\
= & \min _{x \in \mathbb{R}}\left\{x+\frac{1}{F_{X}\left(\zeta_{X}^{-}(\alpha)\right)} \int_{-\infty}^{W_{0}-x} F_{X}(t) d t\right\} \\
\leq & \min _{x \in \mathbb{R}}\left\{x+\frac{1}{\alpha} \int_{-\infty}^{W_{0}-x} F_{Y}(t) d t\right\} \\
= & \min _{x \in \mathbb{R}}\left\{x+\frac{1}{F_{Y}\left(\zeta_{Y}^{-}(\alpha)\right)} \int_{-\infty}^{W_{0}-x} F_{Y}(t) d t\right\} \\
= & \mathrm{C}-\operatorname{VaR}_{Y}(\alpha)
\end{aligned}
$$


since by Lemma 1-a), $F_{X}\left(\zeta_{X}^{-}(\alpha)\right) \geq \alpha$ for all $\alpha$.

So, for atomless random variables with continuum supports, the VaR as a measure of downside risk is stronger than the $\mathrm{C}-\mathrm{VaR}$ and $\mathrm{c}^{*}$-VaR. It will become evident from the subsections below that the converse of the above two relationships are, in general, not valid.

\subsection{SSD and C-VaR}

We can further establish the link between SSD and the conditional-VaR as a measure of risk. To do this, we examine two extreme cases: i) random payoffs with atomless continuum supports; and ii) random payoffs with finite or countable infinite supports.

\subsubsection{The Case with Continuum Supports}

For the case when the random payoffs have continuum supports, we have the following observation on the relationship between the C-VaR and SSD:

Theorem 2 For all $X$ and $Y$ that have continuum supports and are atomless and absolutely integrable, it must hold true that

$$
X \stackrel{S S D}{\succeq} Y \Leftrightarrow C-\operatorname{VaR}_{X}(\alpha) \leq C-\operatorname{VaR}_{Y}(\alpha), \forall \alpha \in(0,1] .
$$

Proof. Recall that for any random variable $W$ that has continuum support and is atomless, we have $F_{W}\left(\zeta_{W}^{-}(\alpha)\right) \equiv \alpha$. Suppose $X \stackrel{\text { SSD }}{\succeq} Y$; that is,

$$
\int_{-\infty}^{x}\left\{F_{X}(t)-F_{Y}(t)\right\} d t \leq 0, \forall x \in \mathbb{R} .
$$

With Proposition 1, we have,

$$
\begin{aligned}
& \mathrm{C}-\operatorname{VaR}_{X}(\alpha) \\
= & \min _{x \in \mathbb{R}}\left\{x+\frac{1}{\alpha} \int_{-\infty}^{W_{0}-x} F_{X}(t) d t\right\} \\
\leq & \min _{x \in \mathbb{R}}\left\{x+\frac{1}{\alpha} \int_{-\infty}^{W_{0}-x} F_{Y}(t) d t\right\} \\
= & \mathrm{C}-\operatorname{VaR}_{Y}(\alpha)
\end{aligned}
$$

for all $\alpha \in(0,1]$. This proves the sufficient part of the theorem. 
To prove the converse, suppose $\mathrm{C}-\operatorname{VaR}_{X}(\alpha) \leq \mathrm{C}-\operatorname{VaR}_{Y}(\alpha)$ for all $\alpha \in$ $(0,1]$. This, together with Proposition 1, implies

$$
\begin{aligned}
& \operatorname{VaR}_{X}(\alpha)+\frac{1}{\alpha} \int_{-\infty}^{W_{0}-\operatorname{VaR}_{X}(\alpha)} F_{X}(t) d t \\
= & \min _{x \in \mathbb{R}}\left\{x+\frac{1}{\alpha} \int_{-\infty}^{W_{0}-x} F_{X}(t) d t\right\} \\
\leq & \min _{x \in \mathbb{R}}\left\{x+\frac{1}{\alpha} \int_{-\infty}^{W_{0}-x} F_{Y}(t) d t\right\} \\
\leq & \operatorname{VaR}_{X}(\alpha)+\frac{1}{\alpha} \int_{-\infty}^{W_{0}-\operatorname{VaR}_{X}(\alpha)} F_{Y}(t) d t .
\end{aligned}
$$

With $\operatorname{VaR}_{X}(\alpha)=W_{0}-\zeta_{X}^{-}(\alpha)$, we obtain

$$
\int_{-\infty}^{\zeta_{X}^{-}(\alpha)}\left\{F_{X}(t)-F_{Y}(t)\right\} d t \leq 0, \forall \alpha \in(0,1]
$$

For any arbitrary $x \in \mathbb{R}$, we may set $\alpha=F_{X}(x)$ to the above inequality to obtain

$\int_{-\infty}^{x}\left\{F_{X}(t)-F_{Y}(t)\right\} d t \leq 0$. This enables us to conclude that $X \stackrel{\text { SSD }}{\succeq} Y$.

Remark 8 Together with the basic properties of SSD, Theorem 2 enables us to establish the following link between $C$-VaR and choices made by riskaverse expected-utility investors: For all $X$ and $Y$ that have continuum supports and are atomless and absolutely integrable, it must hold true that

$$
C-\operatorname{VaR}_{X}(\alpha) \leq C-\operatorname{VaR}_{Y}(\alpha), \forall \alpha \in(0,1] \Leftrightarrow E[u(X)] \geq E[u(Y)]
$$

for all increasing and concave utility functions $u \in \mathbb{C}^{1}(\mathbb{R})$ with bounded first-order derivatives.

Remark 9 The equivalence between SSD and the $C$-VaR criteria stated in Theorem 2 is sensible to the distributional assumption on the random payoffs. As illustrated below, the equivalence will, in general, break down when the random payoffs contain atoms, particularly for random payoffs with discrete supports.

\subsubsection{The Case with Discrete Supports}

For r.v.s $X$ and $Y$ with finite or countable infinite supports that are respectively denoted by $\left\{x_{n}\right\}_{n=1}^{\infty}$ and $\left\{y_{n}\right\}_{n=1}^{\infty}$. Here, $x_{n}<x_{n+1}$ and $y_{n}<y_{n+1}$ 
for $n=1,2, \cdots$. Let $C_{n}$ and $D_{n}, n=0,1,2, \cdots$, be, respectively, the corresponding cumulative probabilities for $X$ and $Y$ with $C_{0}=D_{0}=0$. We have:

Theorem 3 For $X$ and $Y$ with finite or countable infinite supports, it must hold true that

(a) $X \stackrel{S S D}{\succeq} Y \Rightarrow C-V a R_{X}\left(D_{n}\right) \leq C-V a R_{Y}\left(D_{n}\right), \forall n=1,2, \cdots$.

(b) $C$-VaR $\operatorname{Va}_{X}\left(C_{n}\right) \leq C-\operatorname{VaR}_{Y}\left(C_{n}\right), \forall n=1,2, \cdots \Rightarrow X \stackrel{S S D}{\succeq} Y$.

Proof. The C-VaR corresponding to the r.v. $X$ is such that, for all $\alpha \in$ $\left(C_{n}, C_{n+1}\right]$,

$$
\mathrm{C}-\operatorname{VaR}_{X}(\alpha)=W_{0}-x_{n+1}+\frac{1}{C_{n+1}} \int_{-\infty}^{x_{n+1}} F_{X}(t) d t ;
$$

that is, the $\mathrm{C}-\mathrm{VaR}$ is piecewise constant in $\alpha$. A similar observation holds true for $\mathrm{C}-\mathrm{VaR}_{Y}(\alpha)$.

Now, to prove (a), suppose $X \stackrel{\text { SSD }}{\succeq} Y$, that is, $\int_{-\infty}^{x}\left\{F_{X}(t)-F_{Y}(t)\right\} d t \leq$ $0, \forall t \in \mathbb{R}$, we have

$$
\begin{aligned}
& \mathrm{C}-\operatorname{VaR}_{X}(\alpha) \\
& \leq W_{0}-\zeta_{Y}^{-}(\alpha)+\frac{1}{F_{X}\left(\zeta_{X}^{-}(\alpha)\right)} \int_{-\infty}^{\zeta_{Y}^{-}(\alpha)} F_{X}(t) d t \\
& \leq W_{0}-\zeta_{Y}^{-}(\alpha)+\frac{1}{F_{X}\left(\zeta_{X}^{-}(\alpha)\right)} \int_{-\infty}^{\zeta_{Y}^{-}(\alpha)} F_{Y}(t) d t \\
& \leq W_{0}-\zeta_{Y}^{-}(\alpha)+\frac{1}{\alpha} \int_{-\infty}^{\zeta_{Y}^{-}(\alpha)} F_{Y}(t) d t
\end{aligned}
$$

in which, the first inequality follows by applying Proposition 1; the second inequality follows by using the assumption of $X \stackrel{\text { SSD }}{ }$; and the third inequality holds true because of Lemma 1-(a). Particularly at $\alpha=D_{n}$, we have $D_{n}=F_{Y}\left(\zeta_{Y}^{-}\left(D_{n}\right)\right)$ and the above inequality leads to $\mathrm{C}^{-\operatorname{VaR}_{X}}\left(D_{n}\right) \leq$ $\mathrm{C}-\operatorname{VaR}_{Y}\left(D_{n}\right)$ which holds true for all $n=1,2, \cdots$. 
To prove (b), suppose $\mathrm{C}-\operatorname{VaR}_{X}(\alpha) \leq \mathrm{C}-\operatorname{VaR}_{Y}(\alpha)$ at $\alpha=F_{X}\left(x_{n}\right)=C_{n}$ for all $n=1,2, \cdots$, we have:

$$
\begin{aligned}
& W_{0}-x_{n}+\frac{1}{C_{n}} \int_{-\infty}^{x_{n}} F_{X}(t) d t \\
\leq & \mathrm{C}-\operatorname{VaR}_{Y}\left(C_{n}\right) \\
\leq & W_{0}-x_{n}+\frac{1}{F_{Y}\left(\zeta_{Y}^{-}\left(C_{n}\right)\right)} \int_{-\infty}^{x_{n}} F_{Y}(t) d t \\
\leq & W_{0}-x_{n}+\frac{1}{C_{n}} \int_{-\infty}^{x_{n}} F_{Y}(t) d t
\end{aligned}
$$

in which, the first inequality is, by assumption, $\mathrm{C}-\operatorname{VaR}_{X}\left(C_{n}\right) \leq \mathrm{C}-\operatorname{VaR}_{Y}\left(C_{n}\right)$; the second inequality holds because of Proposition 1 ; the third inequality is obtained from the result of Lemma 1-(a). This yields

$$
\int_{-\infty}^{x_{n}}\left\{F_{X}(t)-F_{Y}(t)\right\} d t \leq 0, n=1,2, \cdots .
$$

With the validity of the inequalities above, we could further show that

$$
\int_{-\infty}^{x}\left\{F_{X}(t)-F_{Y}(t)\right\} d t \leq 0, \forall x \in \mathbb{R}
$$

that is, $X \stackrel{\text { SSD }}{\succeq} Y$. In fact, suppose that, to the contrary, there exists $x^{*} \in$ $\left(x_{i}, x_{i+1}\right)$ such that $\int_{-\infty}^{x^{*}}\left\{F_{X}(t)-F_{Y}(t)\right\} d t>0$ and $F_{X}\left(x^{*}\right)-F_{Y}\left(x^{*}\right)>0$. With $F_{X}(x) \equiv C_{i}$ on $\left(x_{i}, x_{i+1}\right)$ and with the c.d.f. $F_{Y}(\cdot)$ to be monotonically increasing, we have $F_{X}(t)-F_{Y}(t)>0$ for all $t \in\left(x^{*}, x_{i+1}\right)$. With $\int_{-\infty}^{x_{i+1}}=$ $\int_{-\infty}^{x^{*}}+\int_{x^{*}}^{x_{i+1}}=(+)+(+)>0$, we obtain a contradiction to

$$
\int_{-\infty}^{x_{i+1}}\left\{F_{X}(t)-F_{Y}(t)\right\} d t \leq 0
$$

As a corollary to Theorem 3, we may state without proof the following observation:

Corollary 2 For $X$ and $Y$ with finite or countable infinite supports, if $C_{n}=$ $D_{n}$ for all $n=1,2, \cdots$, then

$$
X \stackrel{S S D}{\succeq} Y \Leftrightarrow C-\operatorname{VaR}_{X}(\alpha) \leq C-\operatorname{VaR}_{Y}(\alpha), \forall \alpha \in(0,1] .
$$


To sum up, we have shown that, for random payoffs $X$ and $Y$ with continuum supports, $X \stackrel{\text { SSD }}{\succeq} Y$ if and only if $Y$ is riskier than $X$ in the sense that $Y$ has a higher $\mathrm{C}$-VaR than $X$ at all levels of risk tolerance $\alpha \in(0,1]$. From Theorem 3, we observe that the equivalence breaks down for general distributions, particularly when the random payoffs have discrete supports. Since $X \stackrel{\text { SSD }}{\succeq} Y$ if and only if all risk-averse expected-utility investors who prefer more to less will unanimously prefer $X$ to $Y$ (see, for example, Wong and Ma 2006), the C-VaR as an objective measure of downside risk is linked but not always equivalent to the unanimous choice by risk-averse expectedutility investors.

\section{$3.3 \quad c^{*}-$ VaR and SSD}

As we have learned from the previous subsection, generally speaking, the equivalence between SSD and C-VaR does not hold in the absolute sense, particularly for random payoffs with discrete distributions. Nevertheless, by virtue of the proof of the equivalence between SSD and C-VaR for payoffs with continuum supports, we can readily establish the following equivalence relationship between $\mathrm{c}^{*}$-VaR, as a modification to C-VaR, and SSD.

Theorem 4 For all $X$ and $Y$ that are absolutely integrable, it must hold true that

$$
X \stackrel{S S D}{\succeq} Y \Leftrightarrow c^{*}-V a R_{X}(\alpha) \leq c^{*}-V_{a} R_{Y}(\alpha), \forall \alpha \in(0,1] .
$$

Proof. The sufficiency proof is the same as the proof of the sufficient part of Theorem 2. The details are thus omitted.

We proceed with the proof of the necessary part of the theorem. Suppose $c^{*}-\operatorname{VaR}_{X}(\alpha) \leq \mathrm{c}^{*}-\operatorname{VaR}_{Y}(\alpha)$ for all $\alpha \in(0,1]$, by Proposition 2 , we have:

$$
\begin{aligned}
& \operatorname{VaR}_{X}(\alpha)+\frac{1}{\alpha} \int_{-\infty}^{W_{0}-\operatorname{VaR}_{X}(\alpha)} F_{X}(t) d t \\
= & \min _{x \in \mathbb{R}}\left\{x+\frac{1}{\alpha} \int_{-\infty}^{W_{0}-x} F_{X}(t) d t\right\} \\
\leq & \min _{x \in \mathbb{R}}\left\{x+\frac{1}{\alpha} \int_{-\infty}^{W_{0}-x} F_{Y}(t) d t\right\} \\
\leq & \operatorname{VaR}_{X}(\alpha)+\frac{1}{\alpha} \int_{-\infty}^{W_{0}-\operatorname{VaR}_{X}(\alpha)} F_{Y}(t) d t ;
\end{aligned}
$$


that is, with $\operatorname{VaR}_{X}(\alpha)=W_{0}-\zeta_{X}^{-}(\alpha)$,

$$
\int_{-\infty}^{\zeta_{X}^{-}(\alpha)}\left\{F_{X}(t)-F_{Y}(t)\right\} d t \leq 0, \forall \alpha \in(0,1]
$$

We divide the real line $\mathbb{R}$ into three segments:

(a) When $x \in \mathbb{R}$ is located within the continuum support of $X$, we may set $\alpha=F_{X}(x)$ for the above inequality to obtain $\int_{-\infty}^{x}\left\{F_{X}(t)-F_{Y}(t)\right\} d t \leq$ 0

(b) At all atomic support points $x_{1}<x_{2}<\cdots$ of $X$, we may set $\alpha=F_{X}\left(x_{n}\right)$ for the above inequality to obtain $\int_{-\infty}^{x_{n}}\left\{F_{X}(t)-F_{Y}(t)\right\} d t \leq 0$ for all $n=1,2, \cdots$;

(c) Let $O=(a, b)$ be a nuclear of $X$. For all $x \in(a, b)$ that contains no support of $X$, we could still prove $\int_{-\infty}^{x}\left\{F_{X}(t)-F_{Y}(t)\right\} d t \leq 0$.

To prove the last assertion (c), suppose, to the contrary, that there exists $x^{*} \in(a, b)$ such that $\int_{-\infty}^{x^{*}}\left\{F_{X}(t)-F_{Y}(t)\right\} d t>0$. With $F_{X}(x) \equiv F_{X}(a)$ on $(a, b)$ and with the c.d.f. $F_{Y}(\cdot)$ to be monotonically increasing, we must have $F_{X}(t)-F_{Y}(t)>0$ for all $t \in\left[x^{*}, b\right)$. With $\int_{-\infty}^{b}=\int_{-\infty}^{x^{*}}+\int_{x^{*}}^{b}=(+)$ $+(+)>0$, we obtain a contradiction to $\int_{-\infty}^{b}\left\{F_{X}(t)-F_{Y}(t)\right\} d t \leq 0$ since $b$ belongs to the support.

Combining (a), (b) and (c) to obtain

$$
\int_{-\infty}^{x}\left\{F_{X}(t)-F_{Y}(t)\right\} d t \leq 0, \forall x \in \mathbb{R} .
$$

So, we have $X \stackrel{\text { SSD }}{\succeq} Y$.

Thus, it is the modified conditional-VaR, $\mathrm{c}^{*}-\mathrm{VaR}$, rather than the C$\mathrm{VaR}$, that displays equivalence to SSD. A similar result was reported without proof by Leitner (2005, Theorem 2.1) with respect to the equivalence between the so-called SSD and the so-called AV@R, a result originally discovered by Föllmer and Schied (2002, Theorem 2.58 and remark 4.38). Regardless of the technical treatment and proofs, Leitner made use of the right-continuously inverse c.d.f. to define AV@R, while in this paper we adopt the left-continuous c.d.f. to define VaR and the corresponding C-VaR and $\mathrm{c}^{*}$-VaR. Section 4.1 below offers some justifications for our favoring the left- rather than the right-continuous inverse to define the VaR along with the corresponding $\mathrm{C}-\mathrm{VaR}$ and $\mathrm{c}^{*}-\mathrm{VaR}{ }^{8}$

\footnotetext{
${ }^{8}$ Acerbi and Tasche (2002) introduce the notions of lower and upper quantiles, which, respectively, refer to the left- and the right-continuously inverse c.d.f.s. Their notion of
} 
Recall that $X \stackrel{\text { SSD }}{\succeq} Y$ is equivalent to all investors with risk-averse expected utility, who prefer more to less, would prefer $X$ to $Y$ (see, for example, Wong and Ma 2006). Now, as a corollary to Theorem 4, we can alternatively say that all investors with monotonic risk-averse expected utility would prefer $X$ to $Y$ whenever $Y$ is riskier than $X$ in the sense that $Y$ has a higher $c^{*}$-VaR than $X$ at all levels of risk tolerance. Put formally, we have:

Corollary 3 For all $X$ and $Y$ that are absolutely integrable, we have

$$
c^{*}-\operatorname{VaR}_{X}(\alpha) \leq c^{*}-\operatorname{VaR}_{Y}(\alpha), \forall \alpha \in(0,1] \Leftrightarrow E[u(X)] \geq E[u(Y)]
$$

for all increasing and concave utility functions $u \in \mathbb{C}^{1}(\mathbb{R})$ with bounded first-order derivatives.

\section{Discussion}

This section focuses on a general discussion of VaR, C-VaR, and $c^{*}-\mathrm{VaR}$ as risk measures. This goes along with a consideration of some alternative risk measures with somewhat weaker behavior assumptions relative to those for VaRs as risk measures.

\subsection{Optimistic vs. Conservative VaR}

We start with the discussion of the $\mathrm{VaR}$ as a measure of downside risk. The $\mathrm{VaR}$ is of critical importance because both $\mathrm{C}-\mathrm{VaR}$ and $\mathrm{c}^{*}$-VaR as defined above are derived from the VaR. Without the solid justification for the VaR defined in Section 1 as a sound measure of downside risk, we would not be able to recommend that investors use the induced conditional-VaR and modified conditional-VaR. Indeed, as also pointed out by Acerbi and Tasche (2002), there is no unique way to define VaR when the random payoffs have discontinuous c.d.f.s. The definition of VaR is particularly relevant when the random payoffs have discrete supports.

First, it is not difficult to show that the $\operatorname{VaR}(\cdot)$ defined in Section 1 satisfies the following desirable property:

$$
F_{W}\left(W_{0}-x\right)<\alpha \text { for all } \alpha \in(0,1] \text { and } x>\operatorname{VaR}(\alpha) ;
$$

an 'expected shortfall' resembles but differs from our conditional VaR. Acerbi and Tasche (2002) concern the coherence (similar to Artzner et al. 1999) of using various quantiles in defining the expected shortfall. No results like our Theorem 4 were reported in their paper. 
that is, the value at risk so defined could indeed be regarded as a critical value at which the probability that the random loss $W_{0}-W$ would take a value strictly greater than the critical value is below the level of risk tolerance $\alpha$. However, as we have learned from Lemma 1, the above inequality would, in general, be violated at $x=\operatorname{VaR}(\alpha)$, that is,

$$
F_{W}\left(W_{0}-\operatorname{VaR}(\alpha)\right)=F_{W}\left(\zeta_{W}^{-}(\alpha)\right) \geq \alpha .
$$

In fact, the inequality may hold true strictly (for some $\alpha$ ) in the situation when the random payoff $W$ contains atoms. In this sense, one may say that $\operatorname{VaR}(\alpha)$ as a measure of downside risk is optimistic, since the probability that the loss would not be less than the critical value $\operatorname{VaR}(\alpha)$ could be strictly greater than the level of risk tolerance $\alpha$.

On the other hand, we may also argue that $\operatorname{VaR}(\alpha)$ as a measure of downside risk is conservative. To illustrate this, we consider the circumstance when the random payoff $W$ contains an open nuclear $O=(a, b)$ on which no probability mass is assigned. That is, $F_{W}(x) \equiv F_{W}(O)$ for all $x \in O$. According to the definition of VaR, with $a=\inf \left\{x: F_{W}(x) \geq F_{W}(O)\right\}$, the value-at-risk at $\alpha=F_{W}(O)$ is thus given by $W_{0}-a$. In addition, since there is no probability mass assigned on $O=(a, b)$, it is equally sensible to take $W_{0}-b$ or any value within $\left[W_{0}-b, W_{0}-a\right]$ as the value-at-risk at the tolerance level $\alpha=F_{W}(O)$. Since the VaR is set to be at the upper bound of the interval at the pre-specified level of risk tolerance, the VaR as a risk measure is thus regarded as being conservative.

Further to the above observations, we may consider the following alternative measure of value-at-risk, denoted by $\mathrm{VaR}_{0}$, relatively less conservative than the original $\operatorname{VaR}(\alpha)$. The $\operatorname{VaR}_{0}$ defined below would assign $W_{0}-b$ rather than $W_{0}-a$ as its critical value at the risk tolerance level $\alpha=F_{W}(O)$.

Definition 3 For all random payoffs $W$ with initial investment $W_{0}$, we define the optimistic VaR for $W$, denoted by $V_{a} R_{0}(\alpha)$, to be the critical value at which the probability that the loss would exceed the critical value is not greater than $\alpha$, that is, for all $\alpha \in(0,1]$,

$$
\operatorname{VaR}_{0}(\alpha) \triangleq \inf \left\{x: F_{W}\left(W_{0}-x\right) \leq \alpha\right\} .
$$

The $\mathrm{VaR}_{0}$ so-defined obviously satisfies the desirable property that

$$
F_{W}\left(W_{0}-x\right) \leq \alpha \text { for all } x>\operatorname{VaR}_{0}(\alpha) .
$$

Similar to VaR, we may express the $\operatorname{VaR}_{0}(\alpha)$ by making use of the rightcontinuously inverse function $\zeta_{W}^{+}(\cdot)$ :

$$
\operatorname{VaR}_{0}(\alpha)=W_{0}-\zeta_{W}^{+}(\alpha), \forall \alpha \in(0,1] .
$$


The statement made in Lemma 2 -a) is equivalent to $\operatorname{VaR}_{0}(\alpha) \leq \operatorname{VaR}(\alpha), \forall \alpha \in$ $(0,1]$. So, $\operatorname{VaR}_{0}(\alpha)$ as a risk measure is relatively less conservative than $\operatorname{VaR}(\alpha)$, since it assigns a lower level of $\mathrm{VaR}$ at all levels of risk tolerance. For the random payoffs with continuum supports and atomless, it is obvious that the two risk measures $\mathrm{VaR}$ and $\mathrm{VaR}_{0}$ provide the same assessment of the downside risk. However, the two risk measures may differ from each other for random payoffs with discrete supports.

To illustrate such a difference, we consider a random variable $W$ with finite or countable infinite support. We have: $\zeta_{W}^{+}\left(C_{n}\right)=x_{n+1}$ and $\operatorname{VaR}_{0}\left(C_{n}\right)=$ $W_{0}-x_{n+1}$, which are in contrast to $\zeta_{W}^{-}\left(C_{n}\right)=x_{n}$ and $\operatorname{VaR}\left(C_{n}\right)=W_{0}-x_{n}$. Moreover, the two risk measures coincide with each other on $\left(C_{n}, C_{n+1}\right)$, that is, $\operatorname{VaR}_{0}(\alpha)=\operatorname{VaR}(\alpha)=W_{0}-x_{n+1}, \forall \alpha \in\left(C_{n}, C_{n+1}\right)$. Thus, we find that $\mathrm{VaR}_{0}$ sets its critical value at $C_{n}$ to be $W_{0}-x_{n+1}$, which is strictly below $W_{0}-x_{n}$. This assessment of risk offered by $\mathrm{VaR}_{0}$ is thus regarded as over-optimistic.

By virtue of the above observations, we are in favor of using the VaR rather than $\mathrm{VaR}_{0}$ as the critical value at all arbitrary levels of risk tolerance. As a result, we decide not to pursue further the properties for the corresponding conditional- $\mathrm{VaR}_{0}$ and $\mathrm{c}^{*}-\mathrm{VaR}_{0}$ resulting from $\mathrm{VaR}_{0}$, even though much of the mathematical properties for these risk measures could be readily established with the help of Lemma 2.

\subsection{Gambler's Ruin and $\alpha^{*}$-VaRs}

We introduce the following thought experiment known as a gambler's ruin problem:

Example 1 (gambler's ruin) A gambler has a stake of $\$ 5,000$ to bet in two mutually exclusive risk prospects $X$ and $Y . X$ is a one-shot fair gamble with equal probability to lose the stake or to double the bet, that is,

$$
X=\{0.5, \$ 0 ; 0.5, \$ 10,000\} .
$$

$Y$ is also a fair gamble, but its payoff follows an exponential distribution with its p.d.f. given by

$$
f_{Y}(x)=\frac{1}{5000} e^{-\frac{x}{5000}}, x \geq 0 .
$$

\footnotetext{
${ }^{9}$ Here, the realization of $Y$ could be regarded as an accumulated outcome of repeatedly betting in infinitesemal fair gambles. The betting stops when the gambler has lost all of his/her initial stake of $\$ 5000$.
} 
The question is: Which risk prospect would the gambler take? Most people would bet on $Y$ rather than $X$.

That people would bet $Y$ over $X$ does not come as a surprise. $X$ is regarded as riskier than $Y$. By betting on $X$, the gambler faces a $50 \%$ probability of losing all his/her original stake of $\$ 5,000$ ! If instead of investing in $Y$, the gambler could end up with a final stake anywhere on $[0,+\infty)$ with an expected final stake of $\$ 5000$. In other words, $Y$ is regarded as less risky because there is zero probability of losing one's initial stake, yet the gambler has a chance to obtain an arbitrary higher final stake. This outcome does not support VaR and $c^{*}-\mathrm{VaR}$ as good risk measures.

To illustrate the above observation, we plot the following equations, which summarize the VaRs and $\mathrm{c}^{*}$-VaRs associated with $X$ and $Y$. In addition, we find that $X$ and $Y$ do not dominate each other according to the first-order or the second-order stochastic dominance.

$$
\begin{aligned}
\operatorname{VaR}_{X}(\alpha) & =\{\$ 5000, \alpha \in(0.0 .5] ;-\$ 5000, \alpha \in(0.5,1]\} ; \\
\mathrm{c}^{*}-\operatorname{VaR}_{X}(\alpha) & =\left\{\$ 5000, \alpha \in(0.0 .5] ; \$ 5000 \times \frac{1-\alpha}{\alpha}, \alpha \in(0.5,1]\right\} ; \\
\operatorname{VaR}_{Y}(\alpha) & =\$ 5000 \times[1+\ln (1-\alpha)], \alpha \in(0,1] ; \text { and } \\
\mathrm{c}^{*}-\operatorname{VaR}_{Y}(\alpha) & =-\$ 5000 \times \frac{(1-\alpha) \ln (1-\alpha)}{\alpha}, \alpha \in(0,1] .
\end{aligned}
$$

Figure 1: Plots of the c.d.f.s of $X$ and $Y$, their cumulative integrals and the corresponding $\mathrm{VaR}$ and $\mathrm{c}^{*}-\mathrm{VaR}$.

figure $=$ example3.eps, height $=8 \mathrm{~cm}$, width $=14 \mathrm{~cm}$

Note: CCDF stands for cumulative integral of c.d.f.

Nevertheless, it is interesting to observe that the decision to choose $Y$ over $X$ is consistent with the following $\alpha^{*}$-VaRs criterion:

Definition 4 For all random payoffs $X$ and $Y$ that involve the same initial investment, we say that $X$ is riskier than $Y$ according to $\alpha^{*}-V a R$ (resp., $\alpha^{*}-C-V a R$ and $\left.\alpha^{*}-c^{*}-V a R\right)$ if

$$
\begin{aligned}
& \operatorname{VaR}_{X}(\alpha)\left(\text { resp., } C-V a R_{X}(\alpha) \text { and } c^{*}-V a R_{X}(\alpha)\right) \\
\geq & \operatorname{VaR}_{Y}(\alpha)\left(\text { resp. }, C-V a R_{Y}(\alpha) \text { and } c^{*}-V R_{Y}(\alpha)\right)
\end{aligned}
$$

for all $\alpha \in\left(0, \alpha^{*}\right]$.

If we set $\alpha^{*}=0.5$ and $1-e^{-1}$, respectively, for the VaR and $c^{*}$-VaR, then $X$ would be regarded as being riskier than $Y$ because 
- $\operatorname{VaR}_{X}(\alpha)>\operatorname{VaR}_{Y}(\alpha), 0<\alpha<0.5$;

- $\mathrm{c}^{*}-\operatorname{VaR}_{X}(\alpha)>\mathrm{c}^{*}-\operatorname{VaR}_{Y}(\alpha)$ for all $0<\alpha<1-e^{-1}$.

On the other hand, it is easy to verify that

- $\operatorname{VaR}_{X}(\alpha)<\operatorname{VaR}_{Y}(\alpha)$ for $\alpha \in\left(0.5,1-e^{-2}\right)$ and $\operatorname{VaR}_{X}(\alpha)>\operatorname{VaR}_{Y}(\alpha)$ for $\alpha \in\left(1-e^{-2}, 1\right]$;

- $\mathrm{c}^{*}-\operatorname{VaR}_{X}(\alpha)<\mathrm{c}^{*}-\operatorname{VaR}_{Y}(\alpha)$ for $\alpha>1-e^{-1}$.

Gamblers' preferences for $Y$ over $X$ support the hypothesis that people are largely concerned that the VaR or $\mathrm{c}^{*}-\mathrm{VaR}$ for $\alpha$ not exceed a certain psychological limit $\alpha^{*}$ of risk tolerance. ${ }^{10}$ So, it is possible to assess the risk of the risky prospects $X$ and $Y$ even when the corresponding random payoffs do not dominate each other by the first-order or second-order stochastic dominance. We may put this alternatively in terms of VaRs. The outcome of the thought experiment suggests that the $\alpha^{*}$-VaRs could constitute sensible measures of the downside risk in comparison with the original VaRs. The latter corresponds to $\alpha^{*}=1$.

Indeed, it seems to be a common practice in risk management that in assessing financial risk, people are not concerned about downside risk at all levels of risk tolerance but only that $\alpha$ not exceed the pre-specified psychological limit, say, $\alpha^{*}<1$. The maximum level of risk tolerance $\alpha^{*}$ may be set at $5 \%, 10 \%$, or higher, depending on the degree of the investor's psychological risk tolerance. Furthermore, different investors may set different levels of maximum risk tolerance. Presumably, the more cautious the investor, the higher the maximum level of risk tolerance $\alpha^{*}$ would be set. ${ }^{11}$

Taking for granted the $\alpha^{*}$-VaRs as risk measures, we may dig further into the implications on the expected utility as a normalized collective behavior assumption for rational investors. The following general remarks are in order here:

\footnotetext{
${ }^{10}$ To rationalize a gambler's choices, the risk tolerance limit $\alpha^{*}$ would be set not to exceed 0.5 and $1-e^{-1}$ for the VaR and $c^{*}-\mathrm{VaR}$, respectively.

${ }^{11}$ Precisely, investor A is said to be more cautious than investor B if B takes all risk prospects $Y$ that are taken by A, but the converse is not true. Suppose A and B assess risk according to the $\alpha^{*}$-VaRs with their limit of risk tolerance respectively given by $\alpha_{A}^{*}$ and $\alpha_{B}^{*}$. Then, by definition, $\mathrm{A}$ is more cautious than $\mathrm{B}$ if and only if $\alpha_{A}^{*}>\alpha_{B}^{*}$, keeping in mind that

$$
\begin{aligned}
\operatorname{VaR}_{X}(\alpha) \geq \operatorname{VaR}_{Y}(\alpha), \forall \alpha \in\left(0, \alpha_{A}^{*}\right] \\
\Rightarrow \quad \operatorname{VaR}_{X}(\alpha) \geq \operatorname{VaR}_{Y}(\alpha), \forall \alpha \in\left(0, \alpha_{B}^{*}\right] .
\end{aligned}
$$
}


a) Similar to VaRs, the $\alpha^{*}$-VaRs as risk measures constitute partial orders in the space of random payoffs. These are weaker than the original VaRs because $[X$ is riskier than $Y$ according to VaRs $]$ implies $[X$ is riskier than $Y$ according to $\alpha^{*}$-VaRs]; but the converse is, in general, not true. Consequently, the $\alpha^{*}$-VaRs as an investment criterion are no longer equivalent to the corresponding SDs' criterion (unless $\alpha^{*}=1$ ).

b) By virtue of the breakdown of the equivalence between $\alpha^{*}$-VaRs and SDs, as investment criteria, the $\alpha^{*}$-VaRs no longer correspond to investment decisions/portfolio choices made by expected-utility investors. For instance, suppose all investors are expected-utility maximizers with welldiversified preferences, and suppose $X$ is assessed to be riskier than $Y$ according to the $\alpha^{*}$-VaRs, we may ask: would all investors choose $Y$ rather than $X$ ? The answer is no unless $\alpha^{*}=1$ for which $Y$ dominates $X$ in the sense of SD. So, generally speaking, even if $X$ is assessed to be riskier than $Y$ according to $\alpha^{*}$-VaRs, some of the expected-utility investors would still choose $X$ over $Y$.

c) Further to b), suppose all investors use $\alpha^{*}$-VaRs to assess the risk exposure of their investments, and suppose their behavior and investment decisions conform to the $\alpha^{*}$-VaRs criterion $\left(\alpha^{*}<1\right)$, that is, investors never take a project assessed to be riskier according to the $\alpha^{*}$-VaR criterion. We may ask: would such behavior conform to the expected utility axiomatized by von Neumann and Morgenstern (1944)? The answer to this, in theory, is not affirmative. If all investors are expected-utility investors, and if the investors' risk preferences are well-diversified, they may not act unanimously as prescribed by the $\alpha^{*}$-VaRs criterion.

Bearing in mind the above observations on $\alpha^{*}$-VaRs and choice behavior of expected-utility investors, one may wish to conduct experimental studies to gather further evidence for or against the expected utility as a normalized collective behavior assumption for rationality. This is, however, not the objective of this paper.

\subsection{MPS and $\mathrm{c}^{*}-\mathrm{VaR}$}

For the second-order stochastic dominance, which is associated with $\mathrm{c}^{*}$-VaR as a risk measure, we consider the following mean-preserving-spread (MPS) and strong-MPS dominance relationships and the resulting risk measures. 
Definition 5 Given two random payoffs $X$ and $Y, Y$ is said to be a meanpreserving-spread (MPS) of $X$ if $Y=X+\varepsilon$ such that $E[\varepsilon]=0$ and $\operatorname{Cov}(X, \varepsilon)=0$. In addition, $Y$ is said to be a strong-MPS of $X$ if $Y=X+\varepsilon$ such that $E[\varepsilon \mid X]=0$.

Under either of the above definitions, random payoff $Y$, as a MPS (or strong-MPS) of $X$, could be regarded as being riskier than $X$ in the sense that $Y$ is more volatile than $X$. The MPS and strong-MPS dominance relationships are, however, not equivalent to each other because $E[\varepsilon \mid X]=0$ implies, but is not always implied by, $E[\varepsilon]=0$ and $\operatorname{Cov}(X, \varepsilon)=0$. In other words, strong-MPS implies MPS, but the converse is, however, not always true. We write $X \stackrel{\text { dist }}{=} Y$ whenever $X$ and $Y$ are identically distributed.

The following relationship between the strong-MPS and the second-order stochastic dominance is well known (see, for example, Huang and Litzenberger 1988, Chapter 2.8) and was originally proved by Rothschild and Stiglitz (1970):

$$
Y \stackrel{\text { dist }}{=} X+\varepsilon, E[\varepsilon \mid X]=0 \Leftrightarrow X \stackrel{\text { SSD }}{\succeq} Y .
$$

The MPS, as a partial order, is weaker than the strong-MPS. Accordingly, the resulting risk measure associated with MPS would, in general, no longer be represented by the $c^{*}-\mathrm{VaR}$. These findings enable us to establish the following links among MPS, strong-MPS, and the $\mathrm{c}^{*}$-VaR as follows:

Theorem 5 For all random payoffs $X$ and $Y$ with $E[X]=E[Y]$, it must hold true that

(i) $Y \stackrel{\text { dist }}{=} X+\varepsilon, E[\varepsilon \mid X]=0 \Leftrightarrow c^{*}-V a R_{X}(\alpha) \leq c^{*}-V a R_{Y}(\alpha), \forall \alpha \in(0,1]$;

(ii) $c^{*}-\operatorname{VaR}_{X}(\alpha) \leq c^{*}-\operatorname{VaR}_{Y}(\alpha), \forall \alpha \in(0,1] \Rightarrow Y \stackrel{\text { dist }}{=} X+\varepsilon$ with $\operatorname{Cov}(X, \varepsilon)=$ 0 .

The following is an illustrative example to show the violation of the converse of (ii), that is, even if $Y$ is a MPS of $X$, there exists $\alpha \in(0,1)$ such that $X$ has a higher $\mathrm{c}^{*}$-VaR than $Y$ at $\alpha$.

Example 2 Let $\Omega=\{(i, j): i \in\{-1,0,1\}, j \in\{-1,0,1\}\}$ be the state space with equal mass on each of the 9 elements (i.e., $p_{i j}=\frac{1}{9}, \forall(i, j) \in \Omega$ ). Let $\epsilon_{1}$ and $\epsilon_{2}$ be two random seed variables on $\Omega$ that are respectively defined by setting $\epsilon_{1}(i, j)=i$ and $\epsilon_{2}(i, j)=j$ for all $(i, j) \in \Omega$. Consider the following random payoffs $Y=250+200 \epsilon_{2}$ and $X=Y+180 \theta \epsilon_{1}-110 \theta \epsilon_{2}$, where $\theta=$ 
$\frac{1.1 \times 2}{1.1^{2}+1.8^{2}} \approx 0.494$. We have $E[X]=E[Y]=250$ and $\operatorname{Cov}(Y-X, X)=0$, that is, $Y$ is a mean-preserving-spread of $X$.

Let $W_{0}=200$ be the initial investment for the risk prospects. We can compute the $c^{*}-V a R$ for each of the two random payoffs at risk tolerance level $\alpha=i / 9, i=1,2, \cdots, 9$ respectively. We obtain:

\begin{tabular}{c|ccccccccc}
$\alpha$ & $1 / 9$ & $2 / 9$ & $3 / 9$ & $4 / 9$ & $5 / 9$ & $6 / 9$ & $7 / 9$ & $8 / 9$ & 1 \\
\hline$c^{*}-V a R_{Y}$ & 150 & 150 & 150 & 100 & 70 & 50 & 7.14 & -25 & -50 \\
$c^{*}-V a R_{X}$ & 184.61 & 140.12 & 106.42 & 81.4 & 55.12 & 28.16 & 4.28 & -88.57 & -110.36
\end{tabular}

From this example, we find that $c^{*}-V_{a} R_{X}(\alpha)>c^{*}-V a R_{Y}(\alpha)$ at $\alpha=1 / 9$ and $c^{*}-\operatorname{VaR}_{X}(\alpha)<c^{*}-\operatorname{VaR}_{Y}(\alpha)$ for all $\alpha=i / 9, i=2, \cdots, 9$. So, even though $Y$ is a mean-preserving-spread of $X, X$ has a higher $c^{*}-V a R$ than $Y$ at $\alpha=1 / 9$.

Alternatively, we may examine the rank between $X$ and $Y$ by an expectedutility investor. Let $u(x)=\ln (x-15)$ be the utility function. We can readily compute the expected utilities for $X$ and $Y$ respectively:

$$
E[u(X)] \approx 4.75<E[u(Y)] \approx 5.03 .
$$

With either of the above two observations, we conclude that $Y$ being a $M P S$ of $X$ does not imply that $X$ dominates $Y$ according to the SSD. Or in terms of MPS and strong-MPS, we have: " $Y$ is a MPS of $X$ " but it does not imply that " $Y$ is identical in distribution to some strong-MPS of $X$."

An investor is said to display MPS-risk-aversion if $X$ is preferred to $Y$ whenever $Y$ is identical in distribution to a MPS of $X$. For the above example, all MPS risk-averse investors would prefer $X$ to $Y$ because $Y$ could be expressed as a MPS of $X$. So, MPS-risk-averse investors would assess risk differently than those expected-utility investors. From the above example, we also see the difference between the MPS and the $\alpha^{*}$-VaR criterion: $X$ has a higher $\mathrm{c}^{*}$-VaR than $Y$ for all $\alpha \leq \alpha^{*}=1 / 9$, while all MPS-risk-averse investors would consider $X$ to be less risky than $Y$.

Choices made by MPS-risk-averse investors are studied by Boyle and Ma (2004) within the standard context of Markowitz (1959). The following observations are documented there:

(a) All MPS-risk-averse investors would optimally choose to invest along Markowitz's efficient frontier;

(b) All portfolios inside the efficient frontier must be expressed as the MPS of some efficient portfolios (see Boyle and Ma, 2004, Proposition 5). 
These results involve no distributional assumptions on the random payoffs and hold true in the general context with a finite number of risky prospects. It also holds true for MPS-risk-averse investors whose preferences, as partial order, may not admit utility representations, and may thus not belong to any mean-variance utility class.

Observations (a) and (b) have strong implications for how MPS-riskaverse investors would assess risk in the context of portfolio choices. Since the efficient frontier is understood to be obtained by minimizing the portfolio variances at all arbitrary given levels of expected payoffs, and since efficient portfolios at arbitrary expected payoffs are preferred by all MPS-risk-averse investors, the standard deviation as an objective risk measure could be regarded as being supported by choices made by the class of MPS-risk-averse rational investors.

Having said this, we must emphasize again that we do not refer to the pair-wise comparison between the risk prospects. Instead, we are talking about an optimal portfolio holding a finite number of risky prospects. In other words, even if pair-wise comparisons between two risky prospects are not always possible under the MPS criterion, the optimal portfolio held by MPS-risk-averse investors must achieve the minimum 'risk' that is measured by the standard deviation, among all other trading strategies achieving the same level of expected payoff as the optimal portfolio.

Also, the last observation on the efficient portfolios being favored by all MPS-risk-averse investors in general is not valid for the class of riskaverse expected-utility investors. Precisely speaking, portfolios favored by all risk-averse expected-utility investors, in general, may not exist. If such a portfolio exists, it must be identical in distribution with some MPS of those other portfolios with the same level of expected payoff and is thus located on the efficient frontier. But not all efficient portfolios would be favored by risk-averse expected-utility investors as seen from the above example. Thus, it is relatively easier to reach an agreement on risk assessment among MPS-risk-averse investors than among risk-averse expected-utility investors.

To sum up, the MPS and strong-MPS dominance relations correspond to different risk measures. The strong-MPS as a partial order is known to be equivalent to the SSD, which, in turn, points to $\mathrm{c}^{*}$-VaR as the resulting risk measure. The MPS as a weaker partial order than the strong-MPS, on the other hand, is found to be linked to some degree to the standard deviation. In the special case when payoffs of all risk prospects are jointly normal-distributed, the two dominance relations, namely, MPS and strongMPS, are equivalent to each other. As a result, both MPS and strong-MPS would point to the standard deviation as the measure of risk. Indeed, it is 
well-known that under the joint normal distributional assumption, $c^{*}$-VaR as a risk measure is equivalent to the standard deviation.

\section{Concluding Remarks}

This paper provides a decision-theoretical foundation for VaR, conditional$\mathrm{VaR}$, and a modified conditional VaR as risk measures. The relevance of these risk measures is found to be closely connected to unanimous choices made by the expected-utility investors. In fact, such relevance could be established for some general non-expected utility functions. These include the betweenness utility functions of Chew (1983) and Dekel (1986), and the rank-dependent anticipated utility functions of Quiggin (1982) and Yaari (1984, 1987). This largely follows the observation on the equivalence between SSD and choices made by those expected or non-expected utility investors.

The limitations of VaRs as risk measures are well recognized. First, in contrast to the standard deviation as a risk measure, VaRs could only provide partial and incomplete assessments of risk. In other words, with VaRs one cannot carry out pair-wise risk comparisons for two arbitrary risky prospects. Second, even though VaRs are found to be relevant for investors who behave according to the prescribed expected or non-expected utility functions, VaRs as risk measures would make sense for investors who do not behave as such. For example, if investors use $\alpha^{*}$-VaRs, say, for $\alpha^{*}=5 \%$, rather than VaRs to assess risk, or if investors assess risk using the MPS criterion rather than the strong-MPS criterion, then VaRs would no longer be regarded as ideal objective risk measures, at least from the decision-theoretical point of view.

We believe that portfolio selection among all possible combinations of various risky prospects, which is more complicated than the pair-wise comparison of risky prospects, constitutes a more relevant context for studying risk management, in particular risk measurement. A general theory of risk measurement in the context of portfolio choices is beyond the scope of this paper. Nevertheless, it constitutes a future research initiative. To conclude, while it is desirable to have an objective measure for assessing risk prospects, such a measure, if it exists, would be controversial given the subjective nature of risk assessment. 


\section{Appendix 1. Left-continuous Inverse c.d.f.}

The following summarizes the properties of the left-continuously inverse c.d.f. function

Lemma 1 The left-continuously inverse function $\alpha \rightarrow \zeta_{W}^{-}(\alpha)$ defined on $(0,1]$ must be monotonically increasing such that

(a) $F_{W}\left(\zeta_{W}^{-}(\alpha)\right) \geq \alpha, \forall \alpha \in(0,1]$;

(b) $\zeta_{W}^{-}\left(F_{W}(x)\right) \leq x, \forall x \in \mathbb{R}$ s.t. $F_{W}(x)>0$; and

(c) $F_{W}(x)=\inf \left\{\alpha \in(0,1]: \zeta_{W}^{-}(\alpha) \geq x\right\}$ for all $x$ s.t. $F_{W}(x)>0$.

Proof. To see the monotonicity, let $\alpha \geq \alpha^{\prime}$ be located in $(0,1]$. We have

$$
\left\{x: F_{W}(x) \geq \alpha\right\} \subseteq\left\{x: F_{W}(x) \geq \alpha^{\prime}\right\} .
$$

This implies

$$
\zeta_{W}^{-}(\alpha)=\inf \left\{x: F_{W}(x) \geq \alpha\right\} \geq \inf \left\{x: F_{W}(x) \geq \alpha^{\prime}\right\}=\zeta_{W}^{-}\left(\alpha^{\prime}\right)
$$

as desired.

To prove (a), for all arbitrary $\alpha \in(0,1]$, we set $x=\zeta_{W}^{-}(\alpha)$ for $(3)$. By the definition of $\zeta_{W}^{-}(\cdot)$, we have $F_{W}\left(\zeta_{W}^{-}(\alpha)+\varepsilon\right)>\alpha$ for all $\varepsilon>0$ if $\alpha<1$, and $F_{W}\left(\zeta_{W}^{-}(\alpha)+\varepsilon\right)=\alpha$ for all $\varepsilon>0$ if $\alpha=1$. Setting $\varepsilon \rightarrow 0$, by the right continuity of the c.d.f., we obtain $F_{W}\left(\zeta_{W}^{-}(\alpha)\right)=\lim _{\varepsilon \rightarrow 0+} F_{W}\left(\zeta_{W}^{-}(\alpha)+\varepsilon\right) \geq \alpha$ as desired.

To prove (b), for all arbitrary $x \in \mathbb{R}$, let $\alpha^{*}=F_{W}(x)>0$. Since $x \in\left\{y: F_{W}(y) \geq \alpha^{*}\right\}$, we obtain

$$
x \geq \zeta_{W}^{-}\left(\alpha^{*}\right)=\inf \left\{y: F_{W}(y) \geq \alpha^{*}\right\} ;
$$

that is, $\zeta_{W}^{-}\left(F_{W}(x)\right) \leq x$.

To prove (c), for all arbitrary $x \in \mathbb{R}$ with $\alpha^{*}=F_{W}(x) \in(0,1]$. We need to show that

$$
\alpha^{*}=\inf \left\{\alpha \in(0,1]: \zeta_{W}^{-}(\alpha) \geq x\right\} .
$$

First, for all $0 \leq \alpha<\alpha^{*}=F_{W}(x)<1$, by the monotonicity of $\zeta_{W}^{-}(\cdot)$, we have: $\zeta_{W}^{-}(\alpha) \leq \zeta_{W}^{-}\left(\alpha^{*}\right) \leq x$. This allows us to conclude that

$$
\inf \left\{\alpha \in(0,1]: \zeta_{W}^{-}(\alpha) \geq x\right\} \geq \alpha^{*} .
$$


Similarly, for all $1 \geq \alpha>\alpha^{*}$, we have $\zeta_{W}^{-}(\alpha) \geq \zeta_{W}^{-}\left(\alpha^{*}\right)$. We can further show that $\zeta_{W}^{-}(\alpha)>x \geq \zeta_{W}^{-}\left(\alpha^{*}\right), \forall \alpha>\alpha^{*}$. Suppose, to the contrary that, $x \geq \zeta_{W}^{-}(\alpha)=\inf \left\{y: F_{W}(y) \geq \alpha\right\}$. For all $\varepsilon>0$, it must hold true that $F_{W}(x+\varepsilon)>\alpha$. Setting $\varepsilon \rightarrow 0$, we obtain $\alpha^{*}=\lim _{\varepsilon \rightarrow 0+} F_{W}(x+\varepsilon) \geq \alpha$ which contradicts $\alpha>\alpha^{*}$. The condition $\zeta_{W}^{-}(\alpha)>x$ for all $\alpha>\alpha^{*}$ suggests that

$$
\inf \left\{\alpha \in(0,1]: \zeta_{W}^{-}(\alpha) \geq x\right\} \leq \alpha^{*} .
$$

Combining inequalities (15) and (16), we obtain

$$
\alpha^{*}=\inf \left\{\alpha \in(0,1]: \zeta_{W}^{-}(\alpha) \geq x\right\} .
$$

This concludes the proof.

Statement (c) in Lemma 1 is an inversion formula for the c.d.f. from the left-continuously inverse function. For the special case in which the random variable $W$ has continuum support and is atomless, its c.d.f. $F_{W}(\cdot)$, which is strictly monotonically increasing on the support of $W$ has a well-defined inverse function $\zeta_{W}^{-}(\cdot)$ on $(0,1]$ so that $F_{W}\left(\zeta_{W}^{-}(\alpha)\right)=\alpha$ for all $\alpha \in(0,1]$ and $\zeta_{W}^{-}\left(F_{W}(x)\right)=x$ for all $x$ in the support of $W$.

In another extreme, we consider a random variable $W$ with finite or countable infinite support, say $W=\left\{p_{1}, x_{1} ; \cdots ; p_{n}, x_{n} ; \cdots\right\}$ with support $x_{1}<x_{2}<\cdots$. In this case, both the c.d.f. $F_{W}(\cdot)$ and the left-continuously inverse function $\zeta_{W}^{-}(\cdot)$ are piecewise step functions. In particular, if we let $C_{n}=\sum_{i=1}^{n} p_{i}$ with $C_{0}=0$, we have: $\zeta_{W}^{-}(\alpha)=x_{n+1}$, for all $n=0,1, \cdots$, and for all $\alpha \in\left(C_{n}, C_{n+1}\right]$. Thus, the function $\zeta_{W}^{-}(\cdot)$ is monotonically increasing and left-continuous. Moreover, for all $n=1,2, \cdots$, we have $\zeta_{W}^{-}\left(F_{W}(x)\right)=x_{n} \leq x, \forall x \in\left[x_{n}, x_{n+1}\right)$ with $\zeta_{W}^{-}\left(F_{W}(x)\right)<x$ unless $x=$ $x_{n}$; and $F_{W}\left(\zeta_{W}^{-}(\alpha)\right)=C_{n+1} \geq \alpha, \forall \alpha \in\left(C_{n}, C_{n+1}\right]$, with $F_{W}\left(\zeta_{W}^{-}(\alpha)\right)>\alpha$ for $\alpha<C_{n+1}$.

\section{Appendix 2. Right-continuous Inverse c.d.f.}

The lemma below summarizes some joint properties of the left- and the right-continuously inverse of the c.d.f.:

Lemma 2 For all random payoffs $W$, the right-continuously inverse function $\zeta_{W}^{+}(\alpha)$ must be increasing; moreover, it must hold true that

a) $\zeta_{W}^{+}(\alpha) \geq \zeta_{W}^{-}(\alpha)$ for all $\alpha \in(0,1]$;

b) $F_{W}\left(\zeta_{W}^{+}(\alpha)\right) \geq F_{W}\left(\zeta_{W}^{-}(\alpha)\right) \geq \alpha$ for all $\alpha \in(0,1]$; 
c) $\zeta_{W}^{+}\left(F_{W}(x)\right) \geq x \geq \zeta_{W}^{-}\left(F_{W}(x)\right)$ for all $x$ such that $F_{W}(x)>0$; and

d) $F_{W}(x)=\sup \left\{\alpha \in(0,1]: \zeta_{W}^{+}(\alpha) \geq x\right\}$ for all $x$ such that $F_{W}(x)>0$.

Proof. The monotonicity follows, obviously, by the definition of the rightcontinuously inverse function.

To prove a), with $\zeta_{W}^{+}(\alpha)=\sup \left\{x: F_{W}(x) \leq \alpha\right\}$, we have, $\zeta_{W}^{+}(\alpha)+\varepsilon \in$ $\left\{x: F_{W}(x)>\alpha\right\}$ for all $\varepsilon>0$.This, in turn, implies

$$
\zeta_{W}^{+}(\alpha)+\varepsilon \geq \inf \left\{x: F_{W}(x) \geq \alpha\right\}=\zeta_{W}^{-}(\alpha) .
$$

Setting $\varepsilon \rightarrow 0_{+}$we obtain $\zeta_{W}^{+}(\alpha) \geq \zeta_{W}^{-}(\alpha)$.

Statement b) follows by utilizing the monotonicity of the c.d.f. $F_{W}(\cdot)$ and Lemma 1.

Again, with Lemma 1, we only need to prove the validity of the first inequality in c): For all arbitrary $x$ s.t. $F_{W}(x)>0$, we set $\alpha^{*}=F_{W}(x)$. Since $x \in\left\{y: F_{W}(y) \leq \alpha^{*}\right\}$, we have $x \leq \sup \left\{y: F_{W}(y) \leq \alpha^{*}\right\}$, that is, $\zeta_{W}^{+}\left(F_{W}(x)\right) \geq x$ as desired.

To prove $\mathrm{d})$, for all arbitrary $x \in \mathbb{R}$ s.t. $\alpha^{*}=F_{W}(x) \in(0,1]$. For $\alpha \in\left[\alpha^{*}, 1\right]$, by the monotonicity of $\zeta_{W}^{+}(\cdot)$, together with Statement c) of this proposition, we have $\zeta_{W}^{+}(\alpha) \geq \zeta_{W}^{+}\left(\alpha^{*}\right) \geq x$. This implies

$$
\sup \left\{\alpha \in(0,1]: \zeta_{W}^{+}(\alpha) \geq x\right\} \leq \alpha^{*} .
$$

On the other hand, $\zeta_{W}^{+}(\cdot) \geq \zeta_{W}^{-}(\cdot)$ implies

$$
\left\{\alpha \in(0,1]: \zeta_{W}^{+}(\alpha) \geq x\right\} \supseteq\left\{\alpha \in(0,1]: \zeta_{W}^{-}(\alpha) \geq x\right\} .
$$

So, it must hold true that

$$
\sup \left\{\alpha \in(0,1]: \zeta_{W}^{+}(\alpha) \geq x\right\} \geq \inf \left\{\alpha \in(0,1]: \zeta_{W}^{-}(\alpha) \geq x\right\} .
$$

Since, by Lemma 1-c), $\alpha^{*}=F_{W}(x)=\inf \left\{\alpha \in(0,1]: \zeta_{W}^{-}(\alpha) \geq x\right\}$, we obtain

$$
\sup \left\{\alpha \in(0,1]: \zeta_{W}^{+}(\alpha) \geq x\right\} \geq \alpha^{*}
$$

Combining inequalities (17) and (18) yields the desired inversion formula for the c.d.f. $F_{W}(\cdot)$ from the right continuous inverse function $\zeta_{W}^{+}(\cdot)$.

\section{References}

[1] Acerbi C. and Tasche D. (2002), "On the coherence of expected shortfall". Journal of Banking \& Finance 26, 1487-1503. 
[2] Alexander, G.J., Baptista, A.M. (2002), "Economic implications of using mean-VaR model for portfolio selection: a comparison with meanvariance analysis". Journal of Economic and Dynamics Control 26, 1159-1193.

[3] Alexander, G.J., Baptista, A.M. (2004), "Comparison of VaR and CVaR constraints on portfolio selection with the mean-variance model". Management Science 50(9), 1261-1273.

[4] Arrow K.: Essays in the Theory of Risk-Bearing. Amsterdam: NorthHolland, 1970.

[5] Artzner P., Delbaen F., Eber J.M., Heath D. (1999), "Coherent measures of risk". Mathematical Finance 9, 203-228.

[6] Boyle, P. P., Ma, C. (2004), "Mean-preserving-spread risk aversion and the CAPM". University of Essex.

[7] Chew, S.H. (1983), "A generalization of quasilinear mean with applications to the measurement of income inequality and decision theory solving the Allais paradox". Econometrica 51, 1065-1092.

[8] Dekel, E. (1986), "An axiomatic characterization of preferences under uncertainty". Journal of Economic Theory 40, 302-318.

[9] Föllmer H. and Schied A.: Stochastic Finance. Berlin: de Gruyter, 2002.

[10] Gotoh J. and Konno H., "Third degree stochastic dominance and meanrisk analysis". Management Science 46, 289-301.

[11] Hadar, J., Russell, W. (1969), "Rules for ordering uncertain prospects". American Economic Review 59 (1), 25-34.

[12] Hardy G.H., Littlewood, J.E., Pólya, G.: Inequalities. Cambridge University Press, Cambridge, MA, 1934.

[13] Hanoch G., Levy, H. (1969), "The efficiency analysis of choices involving risk". Review of Economic Studies 36, 335-346.

[14] Huang, C.F., Litzenberger, R.H.: Foundations for financial economics, New York: North-Holland, 1988.

[15] Jorion, P.: Value-at-Risk: the new benchmark for managing financial risk. McGraw-Hill: New York, 2000. 
[16] Leitner J. (2005), "A short note on second-order stochastic dominance preserving coherent risk measures". Mathematical Finance 15 (4), 649651.

[17] Levy, H. (1992), "Stochastic dominance and expected utility: survey and analysis". Management Science 38, 555-593.

[18] Ma, C.: Advanced asset pricing theory (in progress). WISE, Xiamen University, 2006.

[19] Markowitz, H.M. (1952), "The utility of wealth". Journal of Political Economy 60, 151-156.

[20] Markowitz, H.M.: Portfolio selection. New York: John Wiley and Sons, Inc., 1959.

[21] Marshall, A.W., Olkin, I.: Inequalities: theory of majorization and its applications. Academic Press, San Diego, 1979.

[22] Ogryczak W. and Ruszczyński A. (2002), "Dual stochastic dominance and related mean-risk models". SIAM Journal of Optimization 13, 6078 .

[23] Pflug G. (2000), "Some remarks on the Value-at-Risk and the conditional Value-at-Risk". Probabilistic Constrained Optimization, S.P. Uryasev (ed.): Kluwer Academic Publishers, 272-281.

[24] Pratt J. (1964), "Risk averse in the small and in the large". Econometrica 32, 122-136.

[25] Quiggin, J. (1982), "A theory of anticipated utility". Journal of Economic Behavior and Organization 3, 323-343.

[26] Quirk J.P., Saposnik, R. (1962), "Admissibility and measurable utility functions". Review of Economic Studies 29, 140-146.

[27] Rockafellar R.T., Uryasev, S.P. (2000), "Optimization of conditional Value-at-Risk". Journal of Risk 2, 21-42.

[28] Rockafellar R.T., Uryasev, S.P. (2002), "Conditional Value-at-Risk for general loss distributions". Journal of Banking and Finance 26, 14431471 .

[29] Rothschild, M., Stiglitz, J.E. (1970), "Increasing risk I. a definition". Journal of Economic Theory 2, 225-243. 
[30] Uryasev S. (2000), "Conditional Value-at-Risk: optimization algorithms and applications". Financial Engineering News 14.

[31] von Neumann, J., Morgenstern, O.: Theory of games and economic behavior. Princeton University Press, Princeton NJ, 1944.

[32] Wong, W.K., Ma, C. (2006), "Preferences over Meyer's location-scale family" (conditionally accepted, Economic Theory ).

[33] Yaari, M. (1984), "Risk aversion without diminishing marginal utility". International Center for Economics and Related Disciplines, London.

[34] Yaari, M. (1987), "A dual theory of choice under risk". Econometrica $55,95-115$. 